\title{
Erratum to: XIV Mediterranean Conference on Medical and Biological Engineering and Computing 2016
}

\author{
Efthyvoulos Kyriacou ${ }^{1}$, Stelios Christofides ${ }^{2}$, and Constantinos S. Pattichis ${ }^{3}$ \\ ${ }^{1}$ Department of Computer Science and Engineering, Frederick University, Nicosia, Cyprus \\ ${ }^{2}$ Biomedical Research Foundation, Nicosia, Cyprus \\ ${ }^{3}$ Department of Computer Science, University of Cyprus, Nicosia, Cyprus
}

\section{Erratum to: \\ E. Kyriacou et al. (eds.) \\ XIV Mediterranean Conference on Medical and Biological Engineering and Computing 2016, IFMBE Proceedings 57, DOI: 10.1007/978-3-319-32703-7}

The original version of this book was inadvertently published with an incorrect chapter pagination from chapter 88 and its following chapters have been changed and differ from the first print versions but also the DOIs have been re-assigned. The correct chapter and the DOIs appear here:

\begin{tabular}{|c|c|c|c|}
\hline Chapter title & Author name & $\begin{array}{l}\text { DOI and page ranges } \\
\text { in old-version }\end{array}$ & Current DOI and page ranges \\
\hline $\begin{array}{l}\text { Preliminary Data on the Usability } \\
\text { and Efficacy of an Assistive Device } \\
\text { for the Congenital Central } \\
\text { Hypoventilation } \\
\text { Syndrome: An Observational Study }\end{array}$ & $\begin{array}{l}\text { Emilia Biffi, C. Piazza, F. } \\
\text { Morandi, P. Avantaggiato, } \\
\text { F. Formica, A. Carcano, } \\
\text { R. Borgatti, } \\
\text { and G. Reni }\end{array}$ & - & $\begin{array}{l}\text { DOI: } 10.1007 / 978-3-319- \\
32703-7 \_88 \\
\text { pp. } 451-456\end{array}$ \\
\hline $\begin{array}{l}\text { Design and Implementation of a } \\
\text { Micro-rheometer for POC } \\
\text { Applications }\end{array}$ & $\begin{array}{l}\text { Laura Ortega Tañá, J. Cid, } \\
\text { A.I. Rodríguez Villarreal, } \\
\text { J. Colomer-Farrarons, and } \\
\text { Pere L. Miribel-Català }\end{array}$ & $\begin{array}{l}\text { DOI: } 10.1007 / 978-3-319- \\
32703-7 \_88 \\
\text { pp. } 451-455\end{array}$ & $\begin{array}{l}\text { DOI: } 10.1007 / 978-3-319- \\
32703-7 \_89 \\
\text { pp. } 457-461\end{array}$ \\
\hline $\begin{array}{l}\text { State of the Art and Future } \\
\text { Prospects of Nanotechnologies in } \\
\text { the Field of Brain-Computer } \\
\text { Interfaces }\end{array}$ & $\begin{array}{l}\text { Alkinoos Athanasiou, } \\
\text { Manousos A. Klados, } \\
\text { Alexander Astaras, Nicolas } \\
\text { Foroglou, Ioannis Magras, } \\
\text { and Panagiotis D. Bamidis }\end{array}$ & $\begin{array}{l}\text { DOI: } 10.1007 / 978-3-319- \\
32703-7 \_89 \\
\text { pp. 456-460 }\end{array}$ & $\begin{array}{l}\text { DOI: } 10.1007 / 978-3-319- \\
32703-7 \_90 \\
\text { pp. 462-466 }\end{array}$ \\
\hline $\begin{array}{l}\text { Design and Validation of an Electric } \\
\text { Circuit Phantom for Galvanic } \\
\text { Intrabody Communication }\end{array}$ & $\begin{array}{l}\text { M. Amparo Callejón, } \\
\text { Javier Reina-Tosina, } \\
\text { David Naranjo, and Laura } \\
\text { M. Roa }\end{array}$ & $\begin{array}{l}\text { DOI: } 10.1007 / 978-3-319- \\
32703-7 \_90 \\
\text { pp. } 461-465\end{array}$ & $\begin{array}{l}\text { DOI: } 10.1007 / 978-3-319- \\
32703-7 \_91 \\
\text { pp. } 467-471\end{array}$ \\
\hline
\end{tabular}

The updated original online version for this book can be found at DOI: 10.1007/978-3-319-32703-7

E. Kyriacou et al. (eds.), XIV Mediterranean Conference on Medical and Biological Engineering and Computing 2016,

IFMBE Proceedings 57,

DOI: 10.1007/978-3-319-32703-7_260 


\begin{tabular}{|c|c|c|c|}
\hline $\begin{array}{l}\text { Development of Smart Sock System } \\
\text { for Gate Analysis and Foot Pressure } \\
\text { Control }\end{array}$ & $\begin{array}{l}\text { Alexander Oks, Alexei } \\
\text { Katashev, M. Zadinans, M. } \\
\text { Rancans, and J. Litvak }\end{array}$ & $\begin{array}{l}\text { DOI: } 10.1007 / 978-3-319- \\
32703-7-91 \\
\text { pp. 466-469 }\end{array}$ & $\begin{array}{l}\text { DOI: } 10.1007 / 978-3-319- \\
32703-7 \_92 \\
\text { pp. } 472-475\end{array}$ \\
\hline $\begin{array}{l}\text { A Preliminary Comparison of Two } \\
\text { Different Methods for Objective } \\
\text { Uniformity Evaluation in Diagnostic } \\
\text { Ultrasound Imaging }\end{array}$ & $\begin{array}{l}\text { Andrea Scorza, Silvia } \\
\text { Conforto, Maurizio } \\
\text { Schmid, Daniele Bibbo, } \\
\text { and Salvatore Andrea } \\
\text { Sciuto }\end{array}$ & $\begin{array}{l}\text { DOI: } 10.1007 / 978-3-319- \\
32703-7 \_92 \\
\text { pp. } 470-475\end{array}$ & $\begin{array}{l}\text { DOI: } 10.1007 / 978-3-319- \\
32703-7-93 \\
\text { pp. } 476-481\end{array}$ \\
\hline $\begin{array}{l}\text { Towards Optically Induced } \\
\text { Semiconductor Human Exhalation } \\
\text { Gas Sensor }\end{array}$ & $\begin{array}{l}\text { Yuri Dekhtyar, Margarita } \\
\text { Selutina, Maksims } \\
\text { Sneiders, and Uldis Zunda }\end{array}$ & $\begin{array}{l}\text { DOI: } 10.1007 / 978-3-319- \\
32703-7=93 \\
\text { pp. 476-479 }\end{array}$ & $\begin{array}{l}\text { DOI: } 10.1007 / 978-3-319- \\
\text { 32703-7_94 } \\
\text { pp. } 482-485\end{array}$ \\
\hline $\begin{array}{l}\text { An Aggregated Cross-Validation } \\
\text { Framework for Computational } \\
\text { Discovery of Disease-Associative } \\
\text { Genes }\end{array}$ & $\begin{array}{l}\text { Omer Faruk Ogutcen, } \\
\text { Zeliha Gormez, } \\
\text { Muhammad Atif Tahir, and } \\
\text { Huseyin Seker }\end{array}$ & $\begin{array}{l}\text { DOI: } 10.1007 / 978-3-319- \\
32703-7=94 \\
\text { pp. } 483-488\end{array}$ & $\begin{array}{l}\text { DOI: } 10.1007 / 978-3-319- \\
32703-7 \_95 \\
\text { pp. } 489-494\end{array}$ \\
\hline $\begin{array}{l}\text { Exploring the Molecular } \\
\text { Determinants of Tumor-Stroma } \\
\text { Interaction in Non-small Cell Lung } \\
\text { Cancer Through the Utilization of } \\
\text { RNA-seq Data from Lung Biopsies }\end{array}$ & $\begin{array}{l}\text { Georgia Kontogianni, } \\
\text { Olga Papadodima, } \\
\text { Achilleas Mitrakas, Ilias } \\
\text { Maglogiannis, Michael I. } \\
\text { Koukourakis, } \\
\text { Alexandra } \\
\text { Giatromanolaki, and } \\
\text { Aristotelis Chatziioannou }\end{array}$ & $\begin{array}{l}\text { DOI: } 10.1007 / 978-3-319- \\
32703-7=95 \\
\text { pp. 489-493 }\end{array}$ & $\begin{array}{l}\text { DOI: } 10.1007 / 978-3-319- \\
32703-7-96 \\
\text { pp. 495-499 }\end{array}$ \\
\hline $\begin{array}{l}\text { Gene Expression Data Analysis for } \\
\text { Classification of Bipolar Disorders }\end{array}$ & $\begin{array}{l}\text { Valsamo Leska, Ekaterini } \\
\text { S. Bei, Euripides Petrakis, } \\
\text { and Michalis Zervakis }\end{array}$ & $\begin{array}{l}\text { DOI: } 10.1007 / 978-3-319- \\
32703-7=96 \\
\text { pp. 494-500 }\end{array}$ & $\begin{array}{l}\text { DOI: } 10.1007 / 978-3-319- \\
32703-7=97 \\
\text { pp. } 500-506\end{array}$ \\
\hline $\begin{array}{l}\text { Complex Dynamics in Tumor Gene } \\
\text { Regulatory Networks: Oncogenesis } \\
\text { Dynamics Driven } \\
\text { by "Genes Gone Crazy" }\end{array}$ & $\begin{array}{l}\text { George I. Lambrou, Maria } \\
\text { Braoudaki, Panagiotis } \\
\text { Katrakazas, Ioannis } \\
\text { Kouris, Dimitra } \\
\text { Iliopoulou, } \\
\text { Tzortzia Koutsouri, } \\
\text { Ourania Petropoulou, and } \\
\text { Dimitrios-Dionysios } \\
\text { Koutsouris }\end{array}$ & $\begin{array}{l}\text { DOI: } 10.1007 / 978-3-319- \\
32703-7=97 \\
\text { pp. 501-505 }\end{array}$ & $\begin{array}{l}\text { DOI: } 10.1007 / 978-3-319- \\
32703-7-98 \\
\text { pp. } 507-511\end{array}$ \\
\hline $\begin{array}{l}\text { Comparing Genomic Network } \\
\text { Methodologies: A Combined } \\
\text { Approach for Cancer Prognosis }\end{array}$ & $\begin{array}{l}\text { Stayroula Tsakaneli, } \\
\text { Ekaterini S. Bei, and } \\
\text { Michalis Zervakis }\end{array}$ & $\begin{array}{l}\text { DOI: } 10.1007 / 978-3-319- \\
32703-7=98 \\
\text { pp. 506-511 }\end{array}$ & $\begin{array}{l}\text { DOI: } 10.1007 / 978-3-319- \\
32703-7 \_99 \\
\text { pp. } 512-517\end{array}$ \\
\hline $\begin{array}{l}\text { Random Forest in Splice Site } \\
\text { Prediction of Human Genome }\end{array}$ & $\begin{array}{l}\text { Elham Pashaei, Mustafa } \\
\text { Ozen, and Nizamettin } \\
\text { Aydin }\end{array}$ & $\begin{array}{l}\text { DOI: } 10.1007 / 978-3-319- \\
32703-799 \\
\text { pp. } 512-517\end{array}$ & $\begin{array}{l}\text { DOI: } 10.1007 / 978-3-319- \\
32703-7 \_100 \\
\text { pp. } 518-523\end{array}$ \\
\hline $\begin{array}{l}\text { Comparing Model Simulation and } \\
\text { Experimental Results to Study the } \\
\text { Dependence on Shear Stress of NO, } \\
\text { ATP } \\
\text { and ADP Production from } \\
\text { Endothelial Cells }\end{array}$ & $\begin{array}{l}\text { Dov Jaron, Patrick Kirby, } \\
\text { Kenneth A. Barbee, Jaimit } \\
\text { Parikh, and Donald G. } \\
\text { Buerk }\end{array}$ & $\begin{array}{l}\text { DOI: } 10.1007 / 978-3-319- \\
32703-7 \_100 \\
\text { p. } 518\end{array}$ & $\begin{array}{l}\text { DOI: } 10.1007 / 978-3-319- \\
32703-7 \_101 \\
\text { p. } 524\end{array}$ \\
\hline $\begin{array}{l}\text { Variable Target Values Neural } \\
\text { Network for Dealing with } \\
\text { Extremely Imbalanced Datasets }\end{array}$ & $\begin{array}{l}\text { Savvas Karatsiolis and } \\
\text { Christos N. Schizas }\end{array}$ & $\begin{array}{l}\text { DOI: } 10.1007 / 978-3-319- \\
32703-7 \_101 \\
\text { pp. 519-522 }\end{array}$ & $\begin{array}{l}\text { DOI: } 10.1007 / 978-3-319- \\
32703-7 \_102 \\
\text { pp. } 525-528\end{array}$ \\
\hline $\begin{array}{l}\text { Novel Methods for Correcting Next } \\
\text { Generation Sequencing Errors in the } \\
\beta \text { Chain of T Cell Receptors }\end{array}$ & $\begin{array}{l}\text { Chrysi Panopoulou, } \\
\text { Christos Maramis, Nicos } \\
\text { Maglaveras, and Ioanna } \\
\text { Chouvarda }\end{array}$ & $\begin{array}{l}\text { DOI: } 10.1007 / 978-3-319- \\
32703-7 \_102 \\
\text { pp. } 523-528\end{array}$ & $\begin{array}{l}\text { DOI: } 10.1007 / 978-3-319- \\
32703-7 \_103 \\
\text { pp. } 529-534\end{array}$ \\
\hline $\begin{array}{l}\text { Using FPGAs to Accelerate Myers } \\
\text { Bit-Vector Algorithm }\end{array}$ & $\begin{array}{l}\text { Jörn Hoffmann, Dirk } \\
\text { Zeckzer, and Martin } \\
\text { Bogdan }\end{array}$ & $\begin{array}{l}\text { DOI: } 10.1007 / 978-3-319- \\
32703-7 \_103 \\
\text { pp. 529-535 }\end{array}$ & $\begin{array}{l}\text { DOI: } 10.1007 / 978-3-319- \\
32703-7 \_104 \\
\text { pp. 535-541 }\end{array}$ \\
\hline
\end{tabular}




\begin{tabular}{|c|c|c|c|}
\hline $\begin{array}{l}\text { Toxic Activity Evaluation by } \\
\text { Clustering }\end{array}$ & $\begin{array}{l}\text { Mauro Giacomini, Angela } \\
\text { Bisio, Stefano Robaldo, } \\
\text { Carmelina Ruggiero, and } \\
\text { Giuseppe Rauch }\end{array}$ & $\begin{array}{l}\text { DOI: } 10.1007 / 978-3-319- \\
32703-7 \_104 \\
\text { pp. 536-539 }\end{array}$ & $\begin{array}{l}\text { DOI: } 10.1007 / 978-3-319- \\
32703-7-105 \\
\text { pp. } 542-545\end{array}$ \\
\hline $\begin{array}{l}\text { Design, User Experience and } \\
\text { Usability Requirements for NGS } \\
\text { Workflows in Clinical Applications }\end{array}$ & $\begin{array}{l}\text { Heimo Müller, Robert } \\
\text { Reihs, and Kurt Zatloukal }\end{array}$ & $\begin{array}{l}\text { DOI: } 10.1007 / 978-3-319- \\
32703-7=105 \\
\text { pp. } 540-544\end{array}$ & $\begin{array}{l}\text { DOI: } 10.1007 / 978-3-319- \\
32703-7=106 \\
\text { pp. 546-550 }\end{array}$ \\
\hline $\begin{array}{l}\text { Estimation of First-Phase Insulin } \\
\text { Secretion in the Zucker Fatty Rat }\end{array}$ & $\begin{array}{l}\text { Francesco Di Nardo, } \\
\text { Micaela Morettini, Carla } \\
\text { E. Cogo, Emanuela Faelli, } \\
\text { Sandro Fioretti, Laura } \\
\text { Burattini, } \\
\text { and Piero Ruggeri }\end{array}$ & $\begin{array}{l}\text { DOI: } 10.1007 / 978-3-319- \\
32703-7-106 \\
\text { pp. } 545-548\end{array}$ & $\begin{array}{l}\text { DOI: } 10.1007 / 978-3-319- \\
32703-7-107 \\
\text { pp. } 551-554\end{array}$ \\
\hline $\begin{array}{l}\text { The Relative Role of Insulin Action } \\
\text { and Secretion in Experimental } \\
\text { Animal Models of Metabolic } \\
\text { Syndrome }\end{array}$ & $\begin{array}{l}\text { Micaela Morettini, } \\
\text { Francesco Di Nardo, } \\
\text { Carla E. Cogo, Emanuela } \\
\text { Faelli, Sandro Fioretti, } \\
\text { Laura Burattini, } \\
\text { and Piero Ruggeri } \\
\end{array}$ & $\begin{array}{l}\text { DOI: } 10.1007 / 978-3-319- \\
32703-7 \_107 \\
\text { pp. 549-552 }\end{array}$ & $\begin{array}{l}\text { DOI: } 10.1007 / 978-3-319- \\
32703-7-108 \\
\text { pp. 555-558 }\end{array}$ \\
\hline $\begin{array}{l}\text { Multiple Kernel Learning } \\
\text { Algorithms and Their Use in } \\
\text { Biomedical Informatics }\end{array}$ & $\begin{array}{l}\text { Evanthia E. Tripoliti, } \\
\text { Michalis Zervakis, and } \\
\text { Dimitrios I. Fotiadis }\end{array}$ & $\begin{array}{l}\text { DOI: } 10.1007 / 978-3-319- \\
32703-7-108 \\
\text { pp. 553-558 }\end{array}$ & $\begin{array}{l}\text { DOI: } 10.1007 / 978-3-319- \\
32703-7 \_109 \\
\text { pp. 559-564 }\end{array}$ \\
\hline $\begin{array}{l}\text { Evaluation of Time Series } \\
\text { Microarray Data for Dynamic Gene } \\
\text { Regulatory Network Inference }\end{array}$ & $\begin{array}{l}\text { Panagiotis Xenitidis, } I . \\
\text { Seimenis, S. Kakolyris, and } \\
\text { A. Adamopoulos }\end{array}$ & $\begin{array}{l}\text { DOI: } 10.1007 / 978-3-319- \\
32703-7-109 \\
\text { pp. 559-562 }\end{array}$ & $\begin{array}{l}\text { DOI: } 10.1007 / 978-3-319- \\
32703-7-110 \\
\text { pp. } 565-568\end{array}$ \\
\hline $\begin{array}{l}\text { Objective Assessment of Children } \\
\text { with Birth Injuries }\end{array}$ & $\begin{array}{l}\text { Ákos Jobbágy, Judit } \\
\text { Schultheisz, Márk } \\
\text { Horváth, Piroska Bacsó, } \\
\text { Péter Csuhaj, and Hanna } \\
\text { Réfy Vraskó }\end{array}$ & $\begin{array}{l}\text { DOI: } 10.1007 / 978-3-319- \\
32703-7-110 \\
\text { pp. } 565-569\end{array}$ & $\begin{array}{l}\text { DOI: } 10.1007 / 978-3-319- \\
32703-7-111 \\
\text { pp. } 571-575\end{array}$ \\
\hline $\begin{array}{l}\text { Investigation into the Effects of } \\
\text { Different Glenohumeral Center } \\
\text { Estimation Methods on Kinematic } \\
\text { \& Kinetic } \\
\text { Calculations }\end{array}$ & $\begin{array}{l}\text { Darren Dawson, Keith } \\
\text { Bryan, and Ger Kelly }\end{array}$ & $\begin{array}{l}\text { DOI: } 10.1007 / 978-3-319- \\
32703-7-111 \\
\text { pp. } 570-573\end{array}$ & $\begin{array}{l}\text { DOI: } 10.1007 / 978-3-319- \\
32703-7-112 \\
\text { pp. } 576-579\end{array}$ \\
\hline $\begin{array}{l}\text { Biomechanical Features of Running } \\
\text { Gait Data Associated with Iliotibial } \\
\text { Band Syndrome: Discrete Variables } \\
\text { Versus Principal Component } \\
\text { Analysis }\end{array}$ & $\begin{array}{l}\text { Angkoon Phinyomark, } \\
\text { Sean T. Osis, Dylan } \\
\text { Kobsar, Blayne A. } \\
\text { Hettinga, Ryan Leigh, and } \\
\text { Reed Ferber }\end{array}$ & $\begin{array}{l}\text { DOI: } 10.1007 / 978-3-319- \\
32703-7 \_112 \\
\text { pp. 574-579 }\end{array}$ & $\begin{array}{l}\text { DOI: } 10.1007 / 978-3-319- \\
32703-7 \_113 \\
\text { pp. 580-585 }\end{array}$ \\
\hline $\begin{array}{l}\text { Kernel Principal Component } \\
\text { Analysis for Identification of } \\
\text { Between-Group Differences and } \\
\text { Changes in Running } \\
\text { Gait Patterns }\end{array}$ & $\begin{array}{l}\text { Angkoon Phinyomark, } \\
\text { Sean T. Osis, Blayne A. } \\
\text { Hettinga, and Reed Ferber }\end{array}$ & $\begin{array}{l}\text { DOI: } 10.1007 / 978-3-319- \\
32703-7-113 \\
\text { pp. } 580-585\end{array}$ & $\begin{array}{l}\text { DOI: } 10.1007 / 978-3-319- \\
32703-7-114 \\
\text { pp. 586-591 }\end{array}$ \\
\hline $\begin{array}{l}\text { Development and Assessment of a } \\
\text { Physiotherapy System Based on } \\
\text { Serious Games }\end{array}$ & $\begin{array}{l}\text { Ioannis Symeonidis and } \\
\text { Ergina Kavallieratou }\end{array}$ & $\begin{array}{l}\text { DOI: } 10.1007 / 978-3-319- \\
32703-7-114 \\
\text { pp. 586-589 }\end{array}$ & $\begin{array}{l}\text { DOI: } 10.1007 / 978-3-319- \\
32703-7-115 \\
\text { pp. 592-595 }\end{array}$ \\
\hline $\begin{array}{l}\text { Effect of Links' Center of Gravity } \\
\text { Position on the Performance of a } \\
\text { Four-Bar Linkage as an Upper Limb } \\
\text { Rehabilitation Mechanism: A } \\
\text { Parametric Study }\end{array}$ & $\begin{array}{l}\text { Evagoras Xydas, } P . \\
\text { Herodotou, Loucas } S . \\
\text { Louca, and Andreas } \\
\text { Mueller }\end{array}$ & $\begin{array}{l}\text { DOI: } 10.1007 / 978-3-319- \\
32703-7=115 \\
\text { pp. } 590-595\end{array}$ & $\begin{array}{l}\text { DOI: } 10.1007 / 978-3-319- \\
32703-7 \_116 \\
\text { pp. 596-601 }\end{array}$ \\
\hline $\begin{array}{l}\text { An Omnidirectional Platform } \\
\text { Design: Application to Posture } \\
\text { Analysis }\end{array}$ & $\begin{array}{l}\text { Naceur Hedjazi, } \\
\text { Abderraouf Benali, } \\
\text { Mourad Bouzit, and Zohir } \\
\text { Dibi }\end{array}$ & $\begin{array}{l}\text { DOI: } 10.1007 / 978-3-319- \\
32703-7-116 \\
\text { pp. 596-601 }\end{array}$ & $\begin{array}{l}\text { DOI: } 10.1007 / 978-3-319- \\
32703-7-117 \\
\text { pp. } 602-607\end{array}$ \\
\hline
\end{tabular}




\begin{tabular}{|c|c|c|c|}
\hline $\begin{array}{l}\text { Mobility Support System for Elderly } \\
\text { Blind People with a Smart Walker } \\
\text { and a Tactile Map }\end{array}$ & $\begin{array}{l}\text { Miguel Reyes Adame, Jing } \\
\text { Yu, and Knut Moeller }\end{array}$ & $\begin{array}{l}\text { DOI: } 10.1007 / 978-3-319- \\
32703-7 \_117 \\
\text { pp. } 602-607\end{array}$ & $\begin{array}{l}\text { DOI: } 10.1007 / 978-3-319- \\
32703-7 \_118 \\
\text { pp. } 608-613\end{array}$ \\
\hline $\begin{array}{l}\text { Torque Collision Detection with } \\
\text { Experimental Validation for } \\
\text { Protontherapy Positioning Robot }\end{array}$ & $\begin{array}{l}\text { Julien Baumeyer, Vincent } \\
\text { Besnard, Sylvain Miossec, } \\
\text { Cyril Novales, Gérard } \\
\text { Poisson, Pierre Vieyres, } \\
\text { and J. Chemouny }\end{array}$ & $\begin{array}{l}\text { DOI: } 10.1007 / 978-3-319- \\
32703-7 \_118 \\
\text { pp. } 608-613\end{array}$ & $\begin{array}{l}\text { DOI: } 10.1007 / 978-3-319- \\
32703-7 \_119 \\
\text { pp. } 614-619\end{array}$ \\
\hline $\begin{array}{l}\text { Mechanical Stress Regulates Tissue } \\
\text { Oxygenation, Cancer Cell } \\
\text { Proliferation and Drug Delivery } \\
\text { During Progression } \\
\text { of Solid Tumors }\end{array}$ & $\begin{array}{l}\text { Fotios Mpekris, Stelios } \\
\text { Angeli, Athanassios P. } \\
\text { Pirentis, and Triantafyllos } \\
\text { Stylianopoulos }\end{array}$ & $\begin{array}{l}\text { DOI: } 10.1007 / 978-3-319- \\
32703-7 \_119 \\
\text { pp. } 614-617\end{array}$ & $\begin{array}{l}\text { DOI: } 10.1007 / 978-3-319- \\
32703-7 \_120 \\
\text { pp. } 620-623\end{array}$ \\
\hline $\begin{array}{l}\text { Numerical Modeling of the Red } \\
\text { Blood Cell Motion/Deformation in } \\
\text { the Capillary }\end{array}$ & $\begin{array}{l}\text { Kamran Hassani, Alireza } \\
\text { Karimi, and Ali Tavakoli } \\
\text { Golpaygani }\end{array}$ & $\begin{array}{l}\text { DOI: } 10.1007 / 978-3-319- \\
32703-7 \_120 \\
\text { pp. } 618-627\end{array}$ & $\begin{array}{l}\text { DOI: } 10.1007 / 978-3-319- \\
32703-7 \_121 \\
\text { pp. } 624-633\end{array}$ \\
\hline $\begin{array}{l}\text { Shape Analysis of Bicipital } \\
\text { Contraction by Means of RGB-D } \\
\text { Sensor, Parallel Transport and } \\
\text { Trajectory Analysis }\end{array}$ & $\begin{array}{l}\text { Michela Goffredo, Paolo } \\
\text { Piras, Valerio Varano, } \\
\text { Stefano Gabriele, Carmen } \\
\text { D'Anna, and Silvia } \\
\text { Conforto }\end{array}$ & $\begin{array}{l}\text { DOI: } 10.1007 / 978-3-319- \\
32703-7 \_121 \\
\text { pp. } 628-633\end{array}$ & $\begin{array}{l}\text { DOI: } 10.1007 / 978-3-319- \\
32703-7 \_122 \\
\text { pp. 634-639 }\end{array}$ \\
\hline $\begin{array}{l}\text { Artificial Neural-Network EMG } \\
\text { Classifier for Hand Movements } \\
\text { Prediction }\end{array}$ & $\begin{array}{l}\text { Marta Gandolla, Simona } \\
\text { Ferrante, Davide } \\
\text { Baldassini, Michele Cotti } \\
\text { Cottini, Carlo Seneci, } \\
\text { and Alessandra Pedrocchi }\end{array}$ & $\begin{array}{l}\text { DOI: } 10.1007 / 978-3-319- \\
32703-7 \_122 \\
\text { pp. } 634-637\end{array}$ & $\begin{array}{l}\text { DOI: } 10.1007 / 978-3-319- \\
32703-7 \_123 \\
\text { pp. } 640-643\end{array}$ \\
\hline $\begin{array}{l}\text { EMG-Controlled Robotic Hand } \\
\text { Rehabilitation Device for Domestic } \\
\text { Training }\end{array}$ & $\begin{array}{l}\text { Marta Gandolla, Simona } \\
\text { Ferrante, D. Baldassini, } \\
\text { Michele Cotti Cottini, } \\
\text { Carlo Seneci, F. Molteni, } \\
\text { E. Guanziroli, and } \\
\text { Alessandra Pedrocchi }\end{array}$ & $\begin{array}{l}\text { DOI: } 10.1007 / 978-3-319- \\
32703-7 \_123 \\
\text { pp. } 638-642\end{array}$ & $\begin{array}{l}\text { DOI: } 10.1007 / 978-3-319- \\
32703-7 \_124 \\
\text { pp. } 644-648\end{array}$ \\
\hline $\begin{array}{l}\text { Design and Modeling of a Joystick } \\
\text { Control Scheme for an Upper Limb } \\
\text { Powered Exoskeleton }\end{array}$ & $\begin{array}{l}\text { Debora Russo, Emilia } \\
\text { Ambrosini, Stefano } \\
\text { Arrigoni, Francesco } \\
\text { Braghin, and Alessandra } \\
\text { Pedrocchi }\end{array}$ & $\begin{array}{l}\text { DOI: } 10.1007 / 978-3-319- \\
32703-7 \_124 \\
\text { pp. } 643-646\end{array}$ & $\begin{array}{l}\text { DOI: } 10.1007 / 978-3-319- \\
32703-7 \_125 \\
\text { pp. } 649-652\end{array}$ \\
\hline $\begin{array}{l}\text { Prosthetic Pacing Device for } \\
\text { Unilateral Facial Paralysis }\end{array}$ & $\begin{array}{l}\text { Ville Rantanen, Antti } \\
\text { Vehkaoja, Jarmo Verho, } \\
\text { Petr Vesely, Jani } \\
\text { Lylykangas, Mirja Ilves, } \\
\text { Eeva Mäkelä, } \\
\text { Markus Rautiainen, Veikko } \\
\text { Surakka, and Jukka Lekkala }\end{array}$ & $\begin{array}{l}\text { DOI: } 10.1007 / 978-3-319- \\
32703-7 \_125 \\
\text { pp. } 647-652\end{array}$ & $\begin{array}{l}\text { DOI: } 10.1007 / 978-3-319- \\
32703-7 \_126 \\
\text { pp. } 653-658\end{array}$ \\
\hline $\begin{array}{l}\text { Patient-Specific Reconstruction of } \\
\text { Large Bone Defects: Clinical } \\
\text { Success Due to an Integrated } \\
\text { Bioengineering } \\
\text { Workflow }\end{array}$ & $\begin{array}{l}\text { W. Bartels, F. Gelaude, } H . \\
\text { Delport, I. Jonkers, and } \\
\text { Jos Vander Sloten }\end{array}$ & $\begin{array}{l}\text { DOI: } 10.1007 / 978-3-319- \\
32703-7 \_126 \\
\text { pp. } 653-656\end{array}$ & $\begin{array}{l}\text { DOI: } 10.1007 / 978-3-319- \\
32703-7 \_127 \\
\text { pp. } 659-662\end{array}$ \\
\hline $\begin{array}{l}\text { Tracking of MRI Interventional } \\
\text { Devices with Computer-Controlled } \\
\text { Detunable Markers }\end{array}$ & $\begin{array}{l}\text { Junmo An, Xin Liu, } \\
\text { Mahmut Unan, Eftychios } \\
\text { G. Christoforou, Andrew } \\
\text { G. Webb, and Nikolaos V. } \\
\text { Tsekos }\end{array}$ & $\begin{array}{l}\text { DOI: } 10.1007 / 978-3-319- \\
32703-7 \_127 \\
\text { pp. } 657-661\end{array}$ & $\begin{array}{l}\text { DOI: } 10.1007 / 978-3-319- \\
32703-7 \_128 \\
\text { pp. } 663-667\end{array}$ \\
\hline $\begin{array}{l}\text { Learning Probabilistic Features } \\
\text { from EMG Data for Predicting Knee } \\
\text { Abnormalities }\end{array}$ & $\begin{array}{l}\text { Jan Kohlschuetter, Jan } \\
\text { Peters, and Elmar } \\
\text { Rueckert }\end{array}$ & $\begin{array}{l}\text { DOI: } 10.1007 / 978-3-319- \\
32703-7 \_128 \\
\text { pp. } 662-666\end{array}$ & $\begin{array}{l}\text { DOI: } 10.1007 / 978-3-319- \\
32703-7 \_129 \\
\text { pp. } 668-672\end{array}$ \\
\hline
\end{tabular}




\begin{tabular}{|c|c|c|c|}
\hline $\begin{array}{l}\text { A Comparative Study Among } \\
\text { Constraint, Robot-Aided and } \\
\text { Standard Therapies in Upper Limb } \\
\text { Rehabilitation } \\
\text { of Children with Acquired Brain } \\
\text { Injury }\end{array}$ & $\begin{array}{l}\text { Ambra Cesareo, Elena } \\
\text { Beretta, Emilia Biffi, } \\
\text { Sandra Strazzer, and } \\
\text { Gianluigi Reni }\end{array}$ & $\begin{array}{l}\text { DOI: } 10.1007 / 978-3-319- \\
32703-7-129 \\
\text { pp. } 667-672\end{array}$ & $\begin{array}{l}\text { DOI: } 10.1007 / 978-3-319- \\
32703-7=130 \\
\text { pp. } 673-678\end{array}$ \\
\hline $\begin{array}{l}\text { A New Transmission Mechanism } \\
\text { for the Actuation of Manipulators } \\
\text { for Magnetic Resonance Imaging } \\
\text { (MRI) } \\
\text { Guided Interventions }\end{array}$ & $\begin{array}{l}\text { Xin Liu, Daniel Biediger, } \\
\text { Rahul Kopru, Eftychios G. } \\
\text { Christoforou, and Nikolaos } \\
\text { V. Tsekos }\end{array}$ & $\begin{array}{l}\text { DOI: } 10.1007 / 978-3-319- \\
32703-7-130 \\
\text { pp. } 673-678\end{array}$ & $\begin{array}{l}\text { DOI: } 10.1007 / 978-3-319- \\
32703-7 \_131 \\
\text { pp. 679-684 }\end{array}$ \\
\hline $\begin{array}{l}\text { Permeability Prediction of Human } \\
\text { Proximal Femoral Trabeculae in the } \\
\text { Direction of Superior-to-Fovea } \\
\text { Utilizing } \\
\text { Directly Measured Microscopic } \\
\text { Poroelastic Properties }\end{array}$ & $\begin{array}{l}\text { Hunhee Kim, Taekyeong } \\
\text { Lee, Youngho Lee, Jaemin } \\
\text { Kim, Soonmoon Jung, } \\
\text { Dongwook Yang, Tae- } \\
\text { Hong Lim, } \\
\text { and Junghwa Hong }\end{array}$ & $\begin{array}{l}\text { DOI: } 10.1007 / 978-3-319- \\
32703-7 \_131 \\
\text { pp. } 679-681\end{array}$ & $\begin{array}{l}\text { DOI: } 10.1007 / 978-3-319- \\
32703-7=132 \\
\text { pp. } 685-687\end{array}$ \\
\hline $\begin{array}{l}\text { A Mathematical Model of Discharge } \\
\text { Coefficient for Prosthetic Valves' } \\
\text { Performance Evaluation }\end{array}$ & $\begin{array}{l}\text { Giuseppe D'Avenio, Carla } \\
\text { Daniele, and Mauro } \\
\text { Grigioni }\end{array}$ & $\begin{array}{l}\text { DOI: } 10.1007 / 978-3-319- \\
32703-7 \_132 \\
\text { pp. } 682-687\end{array}$ & $\begin{array}{l}\text { DOI: } 10.1007 / 978-3-319- \\
32703-7 \_133 \\
\text { pp. } 688-693\end{array}$ \\
\hline $\begin{array}{l}\text { PIV and CFD Insight into the Hinge } \\
\text { and Near-Hinge Flow Fields of } \\
\text { Bileaflet Mechanical Heart Valves }\end{array}$ & $\begin{array}{l}\text { Giuseppe D'Avenio, } \\
\text { Guanglei Wang, Yan Li, } \\
\text { Dan Rafiroiu, Giorgio De } \\
\text { Angelis, and Mauro } \\
\text { Grigioni }\end{array}$ & $\begin{array}{l}\text { DOI: } 10.1007 / 978-3-319- \\
32703-7-133 \\
\text { pp. } 688-693\end{array}$ & $\begin{array}{l}\text { DOI: } 10.1007 / 978-3-319- \\
32703-7=134 \\
\text { pp. 694-699 }\end{array}$ \\
\hline $\begin{array}{l}\text { A Modular Patient Simulator for } \\
\text { Evaluation of Decision Support } \\
\text { Algorithms in Mechanically } \\
\text { Ventilated Patients }\end{array}$ & $\begin{array}{l}\text { Jörn Kretschmer, Thomas } \\
\text { Lehmann, Daniel } \\
\text { Redmond, Patrick Stehle, } \\
\text { and Knut Möller }\end{array}$ & $\begin{array}{l}\text { DOI: } 10.1007 / 978-3-319- \\
32703-7-134 \\
\text { pp. } 697-702\end{array}$ & $\begin{array}{l}\text { DOI: } 10.1007 / 978-3-319- \\
32703-7=135 \\
\text { pp. } 703-708\end{array}$ \\
\hline $\begin{array}{l}\text { Multimodal Quantitative } \\
\text { Assessment for Pre-operative } \\
\text { Prosthesis Selection in Total Hip } \\
\text { Arthroplasty }\end{array}$ & $\begin{array}{l}\text { Kyle Joseph Edmunds, } \\
\text { Prostur Hermannsson, } \\
\text { Mario Barbato, Iris } \\
\text { Árnadóttir, Magnus K. } \\
\text { Gíslason, } \\
\text { Halldor Jónsson Jr., } \\
\text { Delphine Estournet, and } \\
\text { Paolo Gargiulo }\end{array}$ & $\begin{array}{l}\text { DOI: } 10.1007 / 978-3-319- \\
32703-7-135 \\
\text { pp. 703-708 }\end{array}$ & $\begin{array}{l}\text { DOI: } 10.1007 / 978-3-319- \\
32703-7=136 \\
\text { pp. 709-714 }\end{array}$ \\
\hline $\begin{array}{l}\text { A Novel Non-invasive Electrical } \\
\text { Impedance Skin Scanner for Early } \\
\text { Diagnosis Medical Decision } \\
\text { Support } \\
\text { in Clinical Dermatology } \\
\text { (DermaSense) }\end{array}$ & $\begin{array}{l}\text { Alexander Zogkas, Ines } \\
\text { Kirsanidou, } \\
\text { Chrysovalantis Korfitis, } \\
\text { Christina Kemanetzi, } \\
\text { Elisavet Lazaridou, } \\
\text { and Alexander Astaras } \\
\end{array}$ & $\begin{array}{l}\text { DOI: } 10.1007 / 978-3-319- \\
32703-7 \_136 \\
\text { pp. 709-713 }\end{array}$ & $\begin{array}{l}\text { DOI: } 10.1007 / 978-3-319- \\
32703-7=137 \\
\text { pp. } 715-719\end{array}$ \\
\hline $\begin{array}{l}\text { Robot-Assisted System for Free- } \\
\text { Beam Transoral Laser Microsurgery }\end{array}$ & $\begin{array}{l}\text { Leonardo S. Mattos, Luca } \\
\text { Guastini, Francesco Mora, } \\
\text { Darwin G. Caldwell, and } \\
\text { Giorgio Peretti }\end{array}$ & $\begin{array}{l}\text { DOI: } 10.1007 / 978-3-319- \\
32703-7-137 \\
\text { pp. } 714-719\end{array}$ & $\begin{array}{l}\text { DOI: } 10.1007 / 978-3-319- \\
32703-7=138 \\
\text { pp. } 720-725\end{array}$ \\
\hline $\begin{array}{l}\text { Otosclerosis and Tympanosclerosis } \\
\text { Modeling Using the Finite Element } \\
\text { Method }\end{array}$ & $\begin{array}{l}\text { Konstantinos Plakas, } \\
\text { Panagiotis Katrakazas, K. } \\
\text { Giokas, I. Kouris, } \\
\text { Athanasios Bibas, } \\
\text { and Dimitrios-Dionysios } \\
\text { Koutsouris }\end{array}$ & $\begin{array}{l}\text { DOI: } 10.1007 / 978-3-319- \\
32703-7 \_138 \\
\text { pp. } 720-724\end{array}$ & $\begin{array}{l}\text { DOI: } 10.1007 / 978-3-319- \\
32703-7=139 \\
\text { pp. } 726-730\end{array}$ \\
\hline $\begin{array}{l}\text { Non-invasive Trisomy } 21 \text { Diagnosis } \\
\text { Using Fuzzy Cognitive Maps }\end{array}$ & $\begin{array}{l}\text { Maria Papaioannou, } \\
\text { Costas Neocleous, and } \\
\text { Christos N. Schizas }\end{array}$ & $\begin{array}{l}\text { DOI: } 10.1007 / 978-3-319- \\
32703-7+139 \\
\text { pp. } 725-730\end{array}$ & $\begin{array}{l}\text { DOI: } 10.1007 / 978-3-319- \\
32703-7=140 \\
\text { pp. } 731-736\end{array}$ \\
\hline
\end{tabular}




\begin{tabular}{|c|c|c|c|}
\hline $\begin{array}{l}\text { Mechanical in-Exsufflation } \\
\text { Improves the Breathing Pattern in } \\
\text { Patients with Duchenne Muscular } \\
\text { Dystrophy }\end{array}$ & $\begin{array}{l}\text { Ambra Cesareo, Marika } \\
\text { Santi, Antonella LoMauro, } \\
\text { Emilia Biffi, Maria Grazia } \\
\text { D'Angelo, and Andrea } \\
\text { Aliverti }\end{array}$ & $\begin{array}{l}\text { DOI: } 10.1007 / 978-3-319- \\
32703-7 \_140 \\
\text { pp. } 731-734\end{array}$ & $\begin{array}{l}\text { DOI: } 10.1007 / 978-3-319- \\
32703-7-141 \\
\text { pp. } 737-740\end{array}$ \\
\hline $\begin{array}{l}\text { Eyewatch, an Innovative Adjustable } \\
\text { GDD for the Treatment of } \\
\text { Glaucoma - in Vivo Results }\end{array}$ & $\begin{array}{l}\text { Nikolaos Stergiopulos, } \\
\text { Adan Villamarin, André } \\
\text { Mermoud, Stéphane } \\
\text { Bigler, and Sylvain Roy }\end{array}$ & $\begin{array}{l}\text { DOI: } 10.1007 / 978-3-319- \\
32703-7141 \\
\text { pp. } 735-737\end{array}$ & $\begin{array}{l}\text { DOI: } 10.1007 / 978-3-319- \\
32703-7 \_142 \\
\text { pp. } 741-743\end{array}$ \\
\hline $\begin{array}{l}\text { High Intensity Focused Ultrasound } \\
\text { (HIFU) for Biomedical and } \\
\text { Dentistry Applications }\end{array}$ & $\begin{array}{l}\text { Boo Cheong Khoo, Siew- } \\
\text { Wan Ohl, and Evert } \\
\text { Klaseboer }\end{array}$ & $\begin{array}{l}\text { DOI: } 10.1007 / 978-3-319- \\
32703-7 \_142 \\
\text { pp. } 738-741\end{array}$ & $\begin{array}{l}\text { DOI: } 10.1007 / 978-3-319- \\
32703-7 \_143 \\
\text { pp. } 744-747\end{array}$ \\
\hline $\begin{array}{l}\text { Evaluation of Patient Response to } \\
\text { Neoadjuvant Therapy with the Use } \\
\text { of Dynamic Contrast Enhanced } \\
\text { Ultrasound } \\
\text { (DCEUS): Work in Progress }\end{array}$ & $\begin{array}{l}\text { Charis M. Rousou, } \\
\text { Damianos Christofides, } \\
\text { Petros Polyviou, Yiola } \\
\text { Marcou, Eleni Kakouri, } \\
\text { and Mike A. Averkiou }\end{array}$ & $\begin{array}{l}\text { DOI: } 10.1007 / 978-3-319- \\
32703-7=143 \\
\text { pp. } 742-745\end{array}$ & $\begin{array}{l}\text { DOI: } 10.1007 / 978-3-319- \\
32703-7 \_144 \\
\text { pp. } 748-751\end{array}$ \\
\hline $\begin{array}{l}\text { Fabrication and Characterization of } \\
\text { Anisotropic Silk Fibroin/Gelatin } \\
\text { Scaffolds Using Unidirectional } \\
\text { Freezing }\end{array}$ & $\begin{array}{l}\text { Maria Christine Tankeh } \\
\text { Asuncion, Cho Hong } \\
\text { James Goh, and Siew Lok } \\
\text { Toh }\end{array}$ & $\begin{array}{l}\text { DOI: } 10.1007 / 978-3-319- \\
32703-7 \_144 \\
\text { p. } 746\end{array}$ & $\begin{array}{l}\text { DOI: } 10.1007 / 978-3-319- \\
32703-7 \_145 \\
\text { p. } 752\end{array}$ \\
\hline $\begin{array}{l}\text { Near-Infrared Spectroscopy Based } \\
\text { on Portable Instrument for the } \\
\text { Assessment of Glucose } \\
\text { Concentration: In Vitro Experiments }\end{array}$ & $\begin{array}{l}\text { Stefano Sbrignadello, } \\
\text { Giovanni Pacini, and } \\
\text { Andrea Tura }\end{array}$ & $\begin{array}{l}\text { DOI: } 10.1007 / 978-3-319- \\
32703-7 \quad 145 \\
\text { pp. } 747-751\end{array}$ & $\begin{array}{l}\text { DOI: } 10.1007 / 978-3-319- \\
\text { 32703-7_146 } \\
\text { pp. } 753-757\end{array}$ \\
\hline $\begin{array}{l}\text { When the Intensive Care Ventilator } \\
\text { Technology Reaches the Operating } \\
\text { Room: Advancing Ventilation in } \\
\text { Anesthesia }\end{array}$ & $\begin{array}{l}\text { Miloš Ajčević, } U . \\
\text { Lucangelo, W.A. Zin, and } \\
\text { A. Accardo }\end{array}$ & $\begin{array}{l}\text { DOI: } 10.1007 / 978-3-319- \\
32703-7146 \\
\text { pp. } 752-755\end{array}$ & $\begin{array}{l}\text { DOI: } 10.1007 / 978-3-319- \\
32703-7 \_147 \\
\text { pp. } 758-761\end{array}$ \\
\hline $\begin{array}{l}\text { Optical Rhinometry for Long Term } \\
\text { Monitoring }\end{array}$ & $\begin{array}{l}\text { Shao-Hung Lu, Cheng-Lun } \\
\text { Tsai, and Kang-Ping Lin }\end{array}$ & $\begin{array}{l}\text { DOI: } 10.1007 / 978-3-319- \\
32703-7 \_147 \\
\text { pp. } 756-759\end{array}$ & $\begin{array}{l}\text { DOI: } 10.1007 / 978-3-319- \\
32703-7 \_148 \\
\text { pp. } 762-765\end{array}$ \\
\hline $\begin{array}{l}\text { Detection System of Contrast } \\
\text { Sensitivity for Eyes Based on } \\
\text { Digital Micromirror Device }\end{array}$ & $\begin{array}{l}\text { Yongkai Qian, Zili Cao, } \\
\text { and Bixin Zeng }\end{array}$ & $\begin{array}{l}\text { DOI: } 10.1007 / 978-3-319- \\
32703-7 \_148 \\
\text { pp. } 760-763\end{array}$ & $\begin{array}{l}\text { DOI: } 10.1007 / 978-3-319- \\
32703-7 \_149 \\
\text { pp. } 766-769\end{array}$ \\
\hline $\begin{array}{l}\text { Application of Gamma Criteria for } \\
\text { FIF Therapy for Wide Breast Size } \\
\text { Range }\end{array}$ & $\begin{array}{l}\text { Alvis Bernans and Alexei } \\
\text { Katashev }\end{array}$ & $\begin{array}{l}\text { DOI: } 10.1007 / 978-3-319- \\
32703-7 \_149 \\
\text { pp. } 764-767\end{array}$ & $\begin{array}{l}\text { DOI: } 10.1007 / 978-3-319- \\
32703-7 \_150 \\
\text { pp. } 770-773\end{array}$ \\
\hline $\begin{array}{l}\text { Comparison and Classification of } \\
\text { Acoustic Levels of MRI Sequences }\end{array}$ & $\begin{array}{l}\text { Iveta Bryjová, Jan } \\
\text { Kubicek, and H. Skutova }\end{array}$ & $\begin{array}{l}\text { DOI: } 10.1007 / 978-3-319- \\
32703-7 \_150 \\
\text { pp. } 768-772\end{array}$ & $\begin{array}{l}\text { DOI: } 10.1007 / 978-3-319- \\
32703-7 \_151 \\
\text { pp. } 774-778\end{array}$ \\
\hline $\begin{array}{l}\text { Healthcare Information Systems and } \\
\text { Telemedicine Towards an Open } \\
\text { Archival Information System } \\
\text { Compliant Exchange Format to } \\
\text { Ensure Reproducibility of Assays in } \\
\text { Cancer Care }\end{array}$ & $\begin{array}{l}\text { Felix Engel, Paul Walsh, } \\
\text { Heike Görzig, Holger } \\
\text { Brocks, Dominic } \\
\text { Heutelbeck, Brian Kelly, } \\
\text { M. Fuchs, and Matthias } \\
\text { Hemmje }\end{array}$ & $\begin{array}{l}\text { DOI: } 10.1007 / 978-3-319- \\
32703-7-151 \\
\text { pp. } 775-780\end{array}$ & $\begin{array}{l}\text { DOI: } 10.1007 / 978-3-319- \\
32703-7 \_152 \\
\text { pp. } 781-786\end{array}$ \\
\hline $\begin{array}{l}\text { Data Protection Issues of Integrated } \\
\text { Electronic Health Records (EHR) }\end{array}$ & $\begin{array}{l}\text { Eirini C. Schiza, Georgios } \\
\text { J. Fakas, Constantinos S. } \\
\text { Pattichis, Nicolai Petkov, } \\
\text { and Christos N. Schizas }\end{array}$ & $\begin{array}{l}\text { DOI: } 10.1007 / 978-3-319- \\
32703-7 \_152 \\
\text { pp. } 781-784\end{array}$ & $\begin{array}{l}\text { DOI: } 10.1007 / 978-3-319- \\
32703-7 \_153 \\
\text { pp. } 787-790\end{array}$ \\
\hline $\begin{array}{l}\text { An Ontology Based Scheme for } \\
\text { Formal Care Plan Meta-Description }\end{array}$ & $\begin{array}{l}\text { Eleni Kaldoudi, George } \\
\text { Drosatos, Nick Portokallidis, } \\
\text { and Allan Third }\end{array}$ & $\begin{array}{l}\text { DOI: } 10.1007 / 978-3-319- \\
32703-7-153 \\
\text { pp. } 785-790\end{array}$ & $\begin{array}{l}\text { DOI: } 10.1007 / 978-3-319- \\
32703-7 \_154 \\
\text { pp. } 791-796\end{array}$ \\
\hline $\begin{array}{l}\text { An Architecture for Electronic } \\
\text { Prescribing in Physiotherapy in } \\
\text { Belgium }\end{array}$ & $\begin{array}{l}\text { Ronald Buyl, Sven Van } \\
\text { Laere, and Marc Nyssen }\end{array}$ & $\begin{array}{l}\text { DOI: } 10.1007 / 978-3-319- \\
32703-7 \_154 \\
\text { pp. } 791-795\end{array}$ & $\begin{array}{l}\text { DOI: } 10.1007 / 978-3-319- \\
32703-7 \_155 \\
\text { pp. } 797-801\end{array}$ \\
\hline
\end{tabular}




\begin{tabular}{|c|c|c|c|}
\hline $\begin{array}{l}\text { Cloud-Based Information System } \\
\text { for Blood Donation }\end{array}$ & $\begin{array}{l}\text { Ilias Fotopoulos, Revekka } \\
\text { Palaiologou, Ioannis } \\
\text { Kouris, and Dimitrios } \\
\text { Koutsouris }\end{array}$ & $\begin{array}{l}\text { DOI: } 10.1007 / 978-3-319- \\
32703-7 \_155 \\
\text { pp. } 796-801\end{array}$ & $\begin{array}{l}\text { DOI: } 10.1007 / 978-3-319- \\
32703-7 \_156 \\
\text { pp. } 802-807\end{array}$ \\
\hline $\begin{array}{l}\text { The Development of Medical } \\
\text { Ontology "HoPRO" (Hospital } \\
\text { PRocess Ontology) and the Role of } \\
\text { Ontologies in Multimethodological } \\
\text { SAD Frameworks }\end{array}$ & $\begin{array}{l}\text { Vasilios A. Keramaris and } \\
\text { Konstantinos Danas }\end{array}$ & $\begin{array}{l}\text { DOI: } 10.1007 / 978-3-319- \\
32703-7=156 \\
\text { pp. } 802-807\end{array}$ & $\begin{array}{l}\text { DOI: } 10.1007 / 978-3-319- \\
32703-7 \_157 \\
\text { pp. } 808-813\end{array}$ \\
\hline $\begin{array}{l}\text { Feature Selection in Small } \\
\text { Databases: A Medical-Case Study }\end{array}$ & $\begin{array}{l}\text { Inês Soares, Joana Dias, } \\
\text { Humberto Rocha, Maria } \\
\text { do Carmo Lopes, and } \\
\text { Brígida Ferreira }\end{array}$ & $\begin{array}{l}\text { DOI: } 10.1007 / 978-3-319- \\
32703-7 \_157 \\
\text { pp. } 808-813\end{array}$ & $\begin{array}{l}\text { DOI: } 10.1007 / 978-3-319- \\
32703-7 \_158 \\
\text { pp. } 814-819\end{array}$ \\
\hline $\begin{array}{l}\text { Classification of Undetermined } \\
\text { Deaths by Poisoning: Suicidal or } \\
\text { Unintentional }\end{array}$ & $\begin{array}{l}\text { Monique Frize, Hasmik } \\
\text { Martirosyan, Jenini } \\
\text { Subaskaran, Steven R. } \\
\text { McFaull, Robin Skinner, } \\
\text { and Melinda Tiv }\end{array}$ & $\begin{array}{l}\text { DOI: } 10.1007 / 978-3-319- \\
32703-7 \quad 158 \\
\text { pp. } 814-817\end{array}$ & $\begin{array}{l}\text { DOI: } 10.1007 / 978-3-319- \\
32703-7 \_159 \\
\text { pp. } 820-823\end{array}$ \\
\hline $\begin{array}{l}\text { The Cost of Type II Diabetes } \\
\text { Mellitus: A Machine Learning } \\
\text { Perspective }\end{array}$ & $\begin{array}{l}\text { Ghazaal Sheikhi and } \\
\text { Hakan Altınçay }\end{array}$ & $\begin{array}{l}\text { DOI: } 10.1007 / 978-3-319- \\
32703-7 \_159 \\
\text { pp. } 818-821\end{array}$ & $\begin{array}{l}\text { DOI: } 10.1007 / 978-3-319- \\
32703-7 \_160 \\
\text { pp. } 824-827\end{array}$ \\
\hline $\begin{array}{l}\text { Semi-supervised Self-training } \\
\text { Approaches in Small and } \\
\text { Unbalanced Datasets: Application to } \\
\text { Xerostomia Radiation Side-Effect }\end{array}$ & $\begin{array}{l}\text { Inês Soares, Joana Dias, } \\
\text { Humberto Rocha, Leila } \\
\text { Khouri, Maria do Carmo } \\
\text { Lopes, and Brigida } \\
\text { Ferreira }\end{array}$ & $\begin{array}{l}\text { DOI: } 10.1007 / 978-3-319- \\
32703-7 \_160 \\
\text { pp. } 822-827\end{array}$ & $\begin{array}{l}\text { DOI: } 10.1007 / 978-3-319- \\
32703-7 \_161 \\
\text { pp. } 828-833\end{array}$ \\
\hline $\begin{array}{l}\text { Using Performance Measurement in } \\
\text { Healthcare Analytics }\end{array}$ & $\begin{array}{l}\text { Fadi Louis Nammour, } \\
\text { Nashat Mansour, and } \\
\text { Konstantinos Danas }\end{array}$ & $\begin{array}{l}\text { DOI: } 10.1007 / 978-3-319- \\
32703-7 \_161 \\
\text { pp. } 828-833\end{array}$ & $\begin{array}{l}\text { DOI: } 10.1007 / 978-3-319- \\
32703-7 \_162 \\
\text { pp. } 834-839\end{array}$ \\
\hline $\begin{array}{l}\text { Prediction of Healthcare Associated } \\
\text { Infections in an Intensive Care Unit } \\
\text { Using Machine Learning and Big } \\
\text { Data Tools }\end{array}$ & $\begin{array}{l}\text { Paz Revuelta-Zamorano, } \\
\text { Alberto Sánchez, José Luis } \\
\text { Rojo-Álvarez, Joaquín } \\
\text { Álvarez-Rodríguez, Javier } \\
\text { Ramos-López, and Cristina } \\
\text { Soguero-Ruiz }\end{array}$ & $\begin{array}{l}\text { DOI: } 10.1007 / 978-3-319- \\
32703-7 \_162 \\
\text { pp. } 834-839\end{array}$ & $\begin{array}{l}\text { DOI: } 10.1007 / 978-3-319- \\
32703-7 \_163 \\
\text { pp. } 840-845\end{array}$ \\
\hline $\begin{array}{l}\text { Online Platform and Native Mobile } \\
\text { Apps for Patients Support Programs }\end{array}$ & $\begin{array}{l}\text { Ioannis Demetrakopoulos, } \\
\text { Anastasia Anna Vlachou, } \\
\text { Daniel Moussa, and } \\
\text { Stelios Nikoletopoulos }\end{array}$ & $\begin{array}{l}\text { DOI: } 10.1007 / 978-3-319- \\
32703-7 \_163 \\
\text { p. } 840\end{array}$ & $\begin{array}{l}\text { DOI: } 10.1007 / 978-3-319- \\
\text { 32703-7_164 } \\
\text { p. } 846\end{array}$ \\
\hline $\begin{array}{l}\text { Users' Feedback About the Use of a } \\
\text { Telemedicine System During } \\
\text { Emergencies }\end{array}$ & $\begin{array}{l}\text { Efthyvoulos Kyriacou, } \\
\text { Iolie Nicolaidou, Synesios } \\
\text { Christou, Chrysovalantis } \\
\text { Nikolaou, Rafail } \\
\text { Mavrocheilos, Giorgos } \\
\text { Spyrou, Marios } \\
\text { Neophytou, and Riana } \\
\text { Constantinou }\end{array}$ & $\begin{array}{l}\text { DOI: } 10.1007 / 978-3-319- \\
32703-7 \_164 \\
\text { pp. } 841-843\end{array}$ & $\begin{array}{l}\text { DOI: } 10.1007 / 978-3-319- \\
32703-7 \_165 \\
\text { pp. } 847-849\end{array}$ \\
\hline $\begin{array}{l}\text { The Evaluation of an Electronic } \\
\text { Management System for Rescue } \\
\text { Teams by Nursing Personnel }\end{array}$ & $\begin{array}{l}\text { Efthyvoulos Kyriacou, } \\
\text { Iolie Nicolaidou, Nasia } \\
\text { Theodorou, Maria } \\
\text { Nicolaou, M. Tsimaris, } \\
\text { Chrysovalantis Nikolaou, } \\
\text { Marios Neophytou, and } \\
\text { Riana Constantinou }\end{array}$ & $\begin{array}{l}\text { DOI: } 10.1007 / 978-3-319- \\
32703-7 \_165 \\
\text { pp. } 844-847\end{array}$ & $\begin{array}{l}\text { DOI: } 10.1007 / 978-3-319- \\
32703-7 \_166 \\
\text { pp. } 850-853\end{array}$ \\
\hline $\begin{array}{l}\text { A Body-Worn Multi-parameter } \\
\text { Monitoring Platform for Human } \\
\text { Vital Signs Acquisition }\end{array}$ & $\begin{array}{l}\text { Christoph Beck and Julius } \\
\text { Georgiou }\end{array}$ & $\begin{array}{l}\text { DOI: } 10.1007 / 978-3-319- \\
32703-7 \_166 \\
\text { pp. } 848-852\end{array}$ & $\begin{array}{l}\text { DOI: } 10.1007 / 978-3-319- \\
32703-7 \_167 \\
\text { pp. } 854-858\end{array}$ \\
\hline
\end{tabular}




\begin{tabular}{|c|c|c|c|}
\hline $\begin{array}{l}\text { RFCure: An RFID Based Blood } \\
\text { Bank/Healthcare Information } \\
\text { Management System }\end{array}$ & $\begin{array}{l}\text { Eleftheria Katsiri, } \\
\text { Katerina Pramatari, } \\
\text { Antonis Billiris, Aggelos } \\
\text { Kaiafas, Alexandros } \\
\text { Christodoulakis, and } \\
\text { Haralampos Karanikas }\end{array}$ & $\begin{array}{l}\text { DOI: } 10.1007 / 978-3-319- \\
32703-7 \_167 \\
\text { pp. } 853-858\end{array}$ & $\begin{array}{l}\text { DOI: } 10.1007 / 978-3-319- \\
32703-7 \_168 \\
\text { pp. } 859-864\end{array}$ \\
\hline $\begin{array}{l}\text { Towards the Standardization of } \\
\text { Hypnograms Construction for Sleep } \\
\text { Analysis }\end{array}$ & $\begin{array}{l}\text { Ángel Fernández-Leal, } \\
\text { Vicente Moret-Bonillo, and } \\
\text { Mariano J. Cabrero- } \\
\text { Canosa }\end{array}$ & $\begin{array}{l}\text { DOI: } 10.1007 / 978-3-319- \\
32703-7 \_168 \\
\text { pp. } 859-863\end{array}$ & $\begin{array}{l}\text { DOI: } 10.1007 / 978-3-319- \\
32703-7 \_169 \\
\text { pp. } 865-869\end{array}$ \\
\hline $\begin{array}{l}\text { PDF Report as Container for } \\
\text { Structured Clinical Data }\end{array}$ & $\begin{array}{l}\text { Mario Cesarelli, Maria } \\
\text { Romano, Paolo Bifulco, } \\
\text { Francesco Amato, and } \\
\text { Agostino Natale }\end{array}$ & $\begin{array}{l}\text { DOI: } 10.1007 / 978-3-319- \\
32703-7=169 \\
\text { pp. } 864-867\end{array}$ & $\begin{array}{l}\text { DOI: } 10.1007 / 978-3-319- \\
32703-7 \_170 \\
\text { pp. } 870-873\end{array}$ \\
\hline $\begin{array}{l}\text { Ant-inspired Algorithms in Health } \\
\text { Information System Data Mining, } \\
\text { Classification and Visualization }\end{array}$ & $\begin{array}{l}\text { Miroslav Bursa and Lenka } \\
\text { Lhotska }\end{array}$ & $\begin{array}{l}\text { DOI: } 10.1007 / 978-3-319- \\
32703-7 \_170 \\
\text { pp. } 868-873\end{array}$ & $\begin{array}{l}\text { DOI: } 10.1007 / 978-3-319- \\
32703-7 \_171 \\
\text { pp. } 874-879\end{array}$ \\
\hline $\begin{array}{l}\text { A Touchless Gestural Platform for } \\
\text { the Interaction with the Patients } \\
\text { Data }\end{array}$ & Lucio Tommaso De Paolis & $\begin{array}{l}\text { DOI: } 10.1007 / 978-3-319- \\
32703-7 \_171 \\
\text { pp. } 874-878\end{array}$ & $\begin{array}{l}\text { DOI: } 10.1007 / 978-3-319- \\
32703-7 \_172 \\
\text { pp. } 880-884\end{array}$ \\
\hline $\begin{array}{l}\text { Towards the Creation of a Platform } \\
\text { for the Support of Emergency } \\
\text { Health Care Services }\end{array}$ & $\begin{array}{l}\text { Synesios Christou, George } \\
\text { Hadjichristofi, Riana } \\
\text { Constantinou, and } \\
\text { Efthyvoulos Kyriacou }\end{array}$ & $\begin{array}{l}\text { DOI: } 10.1007 / 978-3-319- \\
32703-7 \_172 \\
\text { pp. } 879-882\end{array}$ & $\begin{array}{l}\text { DOI: } 10.1007 / 978-3-319- \\
32703-7 \_173 \\
\text { pp. } 885-888\end{array}$ \\
\hline $\begin{array}{l}\text { An Age Simulated Virtual } \\
\text { Environment for Improving Elderly } \\
\text { Wellbeing }\end{array}$ & $\begin{array}{l}\text { Christina Zavlanou and } \\
\text { Andreas Lanitis }\end{array}$ & $\begin{array}{l}\text { DOI: } 10.1007 / 978-3-319- \\
32703-7 \_173 \\
\text { pp. } 885-890\end{array}$ & $\begin{array}{l}\text { DOI: } 10.1007 / 978-3-319- \\
32703-7 \_174 \\
\text { pp. } 891-896\end{array}$ \\
\hline $\begin{array}{l}\text { KeepWell: A Generic Platform for } \\
\text { the Self-Management of Chronic } \\
\text { Conditions }\end{array}$ & $\begin{array}{l}\text { Timothy Patterson, } \\
\text { Federico Cruciani, Ian } \\
\text { Cleland, Chris D. Nugent, } \\
\text { Norman D. Black, Paul J. } \\
\text { McCullagh, Huiru Zheng, } \\
\text { Mark P. Donnelly, } \\
\text { Suzanne McDonough, and } \\
\text { Adele Boyd }\end{array}$ & $\begin{array}{l}\text { DOI: } 10.1007 / 978-3-319- \\
32703-71174 \\
\text { pp. } 891-896\end{array}$ & $\begin{array}{l}\text { DOI: } 10.1007 / 978-3-319- \\
32703-7 \_175 \\
\text { pp. } 897-902\end{array}$ \\
\hline $\begin{array}{l}\text { ICT Systems and Services for } \\
\text { Ageing Well: Identification and } \\
\text { Assessment of an Important Set } \\
\text { (Package) of ICT Services for } \\
\text { Active Ageing and Independent } \\
\text { Living }\end{array}$ & $\begin{array}{l}\text { Christophoros } \\
\text { Christophorou, } \\
\text { Dimosthenis Georgiadis, } \\
\text { Panayiotis Andreou, } \\
\text { Styliani Kleanthous, } \\
\text { Donato M. Cereghetti, } \\
\text { Judith Meijers, Eleni } \\
\text { Christodoulou, and } \\
\text { George Samaras } \\
\end{array}$ & $\begin{array}{l}\text { DOI: } 10.1007 / 978-3-319- \\
32703-7 \_175 \\
\text { pp. } 897-902\end{array}$ & $\begin{array}{l}\text { DOI: } 10.1007 / 978-3-319- \\
\text { 32703-7_176 } \\
\text { pp. 903-908 }\end{array}$ \\
\hline $\begin{array}{l}\text { Does the Use of a Serious Game and } \\
\text { the Grip-Ball Decrease Discomfort } \\
\text { in Older People When Assessing } \\
\text { Maximal Grip-Strength? }\end{array}$ & $\begin{array}{l}\text { Aly Chkeir, Dimitri } \\
\text { Voilmy, Jacques Duchêne, } \\
\text { and David J. Hewson }\end{array}$ & $\begin{array}{l}\text { DOI: } 10.1007 / 978-3-319- \\
32703-7=176 \\
\text { pp. } 903-906\end{array}$ & $\begin{array}{l}\text { DOI: } 10.1007 / 978-3-319- \\
\text { 32703-7_177 } \\
\text { pp. 909-912 }\end{array}$ \\
\hline $\begin{array}{l}\text { State of Alertness During Simulated } \\
\text { Driving Tasks }\end{array}$ & $\begin{array}{l}\text { Carmen D’Anna, Daniele } \\
\text { Bibbo, Maurizio Bertollo, } \\
\text { Selenia di Fronso, Silvia } \\
\text { Comani, Maria Rosaria } \\
\text { De Blasiis, Valerio } \\
\text { Veraldi, Michela Goffredo, } \\
\text { and Silvia Conforto }\end{array}$ & $\begin{array}{l}\text { DOI: } 10.1007 / 978-3-319- \\
32703-7 \_177 \\
\text { pp. } 907-912\end{array}$ & $\begin{array}{l}\text { DOI: } 10.1007 / 978-3-319- \\
\text { 32703-7_178 } \\
\text { pp. 913-918 }\end{array}$ \\
\hline
\end{tabular}




\begin{tabular}{|c|c|c|c|}
\hline $\begin{array}{l}\text { A Robotic Cloud Ecosystem for } \\
\text { Elderly Care and Ageing Well: The } \\
\text { GrowMeUp Approach }\end{array}$ & $\begin{array}{l}\text { Dimosthenis Georgiadis, } \\
\text { Christophoros } \\
\text { Christophorou, Styliani } \\
\text { Kleanthous, Panayiotis } \\
\text { Andreou, Luis Santos, } \\
\text { Eleni Christodoulou, and } \\
\text { George Samaras }\end{array}$ & $\begin{array}{l}\text { DOI: } 10.1007 / 978-3-319- \\
32703-7-178 \\
\text { pp. 913-918 }\end{array}$ & $\begin{array}{l}\text { DOI: } 10.1007 / 978-3-319- \\
32703-7 \_179 \\
\text { pp. 919-924 }\end{array}$ \\
\hline $\begin{array}{l}\text { The First e-Learning Materials in } \\
\text { Medical Physics - Development, } \\
\text { Practical Application and Impact - } \\
\text { An Overview of } 7 \text { International } \\
\text { Projects }\end{array}$ & Slavik Tabakov & $\begin{array}{l}\text { DOI: } 10.1007 / 978-3-319- \\
32703-7 \_179 \\
\text { pp. 921-925 }\end{array}$ & $\begin{array}{l}\text { DOI: } 10.1007 / 978-3-319- \\
32703-7=180 \\
\text { pp. } 927-931\end{array}$ \\
\hline $\begin{array}{l}\text { A Technological Platform to } \\
\text { Support Education in Regional } \\
\text { Anaesthesia with Patient-Specific } \\
\text { Virtual Physiological Human } \\
\text { (VPH)-Based Models }\end{array}$ & $\begin{array}{l}\text { Georgios Christodoulakis, } \\
\text { K. Marias, G. Notas, } N . \\
\text { Kampanis, and S. } \\
\text { Sfakianakis }\end{array}$ & $\begin{array}{l}\text { DOI: } 10.1007 / 978-3-319- \\
32703-7-180 \\
\text { pp. } 926-929\end{array}$ & $\begin{array}{l}\text { DOI: } 10.1007 / 978-3-319- \\
32703-7 \_181 \\
\text { pp. 932-935 }\end{array}$ \\
\hline $\begin{array}{l}\text { Prenatal Diagnosis of Aneuploidy } \\
\text { Using Artificial Neural Networks in } \\
\text { Relation to Health Economics }\end{array}$ & $\begin{array}{l}\text { Andreas C. Neocleous, } \\
\text { Costas K. Neocleous, } \\
\text { Nicolai Petkov, Kypros H. } \\
\text { Nicolaides, and Christos } \\
\text { N. Schizas }\end{array}$ & $\begin{array}{l}\text { DOI: } 10.1007 / 978-3-319- \\
32703-7 \_181 \\
\text { pp. 930-934 }\end{array}$ & $\begin{array}{l}\text { DOI: } 10.1007 / 978-3-319- \\
32703-7-182 \\
\text { pp. 936-940 }\end{array}$ \\
\hline $\begin{array}{l}\text { Metrological Reliability and } \\
\text { Electrical Safety: A Case Study on } \\
\text { Electrosurgical Equipment }\end{array}$ & $\begin{array}{l}\text { Ali Ramezani, Ali Tavakoli } \\
\text { Golpaygani, and } \\
\text { Mohammad Mehdi } \\
\text { Movahedi }\end{array}$ & $\begin{array}{l}\text { DOI: } 10.1007 / 978-3-319- \\
32703-7 \_182 \\
\text { pp. 935-939 }\end{array}$ & $\begin{array}{l}\text { DOI: } 10.1007 / 978-3-319- \\
32703-7 \_183 \\
\text { pp. } 941-945\end{array}$ \\
\hline $\begin{array}{l}\text { Interactive Application for } \\
\text { Simulation of Each Type of } \\
\text { Defibrillation Impulses by Using } \\
\text { LabVIEW }\end{array}$ & $\begin{array}{l}\text { Lukas Peter and Radek } \\
\text { Osmancik }\end{array}$ & $\begin{array}{l}\text { DOI: } 10.1007 / 978-3-319- \\
32703-7-183 \\
\text { pp. } 940-943\end{array}$ & $\begin{array}{l}\text { DOI: } 10.1007 / 978-3-319- \\
32703-7-184 \\
\text { pp. } 946-949\end{array}$ \\
\hline $\begin{array}{l}\text { Radiation Protection - A Global } \\
\text { View. A New IOMP-IRPA } \\
\text { Initiative }\end{array}$ & M. Stoeva and R. Vetter & $\begin{array}{l}\text { DOI: } 10.1007 / 978-3-319- \\
32703-7 \_184 \\
\text { pp. } 944-947\end{array}$ & $\begin{array}{l}\text { DOI: } 10.1007 / 978-3-319- \\
32703-7=185 \\
\text { pp. } 950-953\end{array}$ \\
\hline $\begin{array}{l}\text { Software Solution for Tracking } \\
\text { Inspection Processes of Medical } \\
\text { Devices from Legal Metrology } \\
\text { System }\end{array}$ & $\begin{array}{l}\text { Lejla Gurbeta, Dijana } \\
\text { Sejdinović, Berina Alić, } \\
\text { Layla Abd El-Ilah, Almir } \\
\text { Badnjević, and Emir Žunić }\end{array}$ & $\begin{array}{l}\text { DOI: } 10.1007 / 978-3-319- \\
32703-7 \_185 \\
\text { pp. } 951-955\end{array}$ & $\begin{array}{l}\text { DOI: } 10.1007 / 978-3-319- \\
32703-7 \_186 \\
\text { pp. 957-961 }\end{array}$ \\
\hline $\begin{array}{l}\text { Implementing Audio and Visual } \\
\text { Alarms to an Intensive Care } \\
\text { Cerebral Function Monitor: A } \\
\text { Standards Compliance Case Study }\end{array}$ & $\begin{array}{l}\text { Angelo Bernardo B. Souza, } \\
\text { Paulo Danilo Farina Jr., } \\
\text { Mauricio Cagy, and } \\
\text { Antonio Fernando C. } \\
\text { Infantosi }\end{array}$ & $\begin{array}{l}\text { DOI: } 10.1007 / 978-3-319- \\
32703-7-186 \\
\text { pp. 956-960 }\end{array}$ & $\begin{array}{l}\text { DOI: } 10.1007 / 978-3-319- \\
32703-7 \_187 \\
\text { pp. 962-966 }\end{array}$ \\
\hline $\begin{array}{l}\text { Discrete Event Simulation-Based } \\
\text { Approach for Hospital Services } \\
\text { Development and Monitoring }\end{array}$ & $\begin{array}{l}\text { Duccio Cocchi, Roberto } \\
\text { Miniati, Francesco } \\
\text { Frosini, Paolo Avezzano, } \\
\text { Ernesto Iadanza, Matteo } \\
\text { Curiardi, and Andrea } \\
\text { Belardinelli }\end{array}$ & $\begin{array}{l}\text { DOI: } 10.1007 / 978-3-319- \\
32703-7 \_187 \\
\text { pp. 961-964 }\end{array}$ & $\begin{array}{l}\text { DOI: } 10.1007 / 978-3-319- \\
32703-7-188 \\
\text { pp. } 967-970\end{array}$ \\
\hline $\begin{array}{l}\text { Assessing the Impact of a } \\
\text { CIS/PACS Technology for a } \\
\text { Cardiology Department Using QFD } \\
\text { Methodology }\end{array}$ & $\begin{array}{l}\text { Alessio Luschi, Laura } \\
\text { Caltagirone, Claudio } \\
\text { Mondovecchio, Roberto } \\
\text { Miniati, and Ernesto Iadanza }\end{array}$ & $\begin{array}{l}\text { DOI: } 10.1007 / 978-3-319- \\
32703-7-188 \\
\text { pp. } 965-968\end{array}$ & $\begin{array}{l}\text { DOI: } 10.1007 / 978-3-319- \\
32703-7 \_189 \\
\text { pp. } 971-974\end{array}$ \\
\hline $\begin{array}{l}\text { The Benefit of in-Hospital Clinical } \\
\text { Engineer Services for Medical } \\
\text { Devices Maintenance }\end{array}$ & $\begin{array}{l}\text { Mei-Fen Chen, Shiang-Lin } \\
\text { Chu, Jen-Kai Lee, Fun- } \\
\text { Hsiang Lin, Cheng-Lung } \\
\text { Tsai, Tsai Kao, and Kang- } \\
\text { Ping Lin }\end{array}$ & $\begin{array}{l}\text { DOI: } 10.1007 / 978-3-319- \\
32703-7 \_189 \\
\text { pp. 969-972 }\end{array}$ & $\begin{array}{l}\text { DOI: } 10.1007 / 978-3-319- \\
32703-7-190 \\
\text { pp. 975-978 }\end{array}$ \\
\hline
\end{tabular}




\begin{tabular}{|c|c|c|c|}
\hline $\begin{array}{l}\text { Mobile Health Applications: } \\
\text { Design, Regulation and Assessment }\end{array}$ & $\begin{array}{l}\text { Mary Marinou and Nicolas } \\
\text { Pallikarakis }\end{array}$ & $\begin{array}{l}\text { DOI: } 10.1007 / 978-3-319- \\
32703-7 \_190 \\
\text { pp. 973-976 }\end{array}$ & $\begin{array}{l}\text { DOI: } 10.1007 / 978-3-319- \\
32703-7 \_191 \\
\text { pp. 979-982 }\end{array}$ \\
\hline $\begin{array}{l}\text { Computer Aided Multispectral } \\
\text { Ultrasound Diagnostics Brain } \\
\text { Health Monitoring System Based on } \\
\text { Acoustocerebrography }\end{array}$ & $\begin{array}{l}\text { Martin Bogdan, Adam } \\
\text { Kolany, Ulrike Weber, } \\
\text { Romy Elze, and Miroslaw } \\
\text { Wrobel }\end{array}$ & $\begin{array}{l}\text { DOI: } 10.1007 / 978-3-319- \\
32703-7 \_191 \\
\text { pp. } 977-981\end{array}$ & $\begin{array}{l}\text { DOI: } 10.1007 / 978-3-319- \\
\text { 32703-7_192 } \\
\text { pp. 983-987 }\end{array}$ \\
\hline $\begin{array}{l}\text { Heuristic Evaluation of a Toolset for } \\
\text { Type } 2 \text { Diabetes Mellitus } \\
\text { Management }\end{array}$ & $\begin{array}{l}\text { Jorge Cancela, Liss } \\
\text { Hernandez, Giuseppe } \\
\text { Fico, and Maria Teresa } \\
\text { Arredondo Waldmeyer }\end{array}$ & $\begin{array}{l}\text { DOI: } 10.1007 / 978-3-319- \\
\text { 32703-7_192 } \\
\text { pp. 982-987 }\end{array}$ & $\begin{array}{l}\text { DOI: } 10.1007 / 978-3-319- \\
\text { 32703-7_193 } \\
\text { pp. 988-993 }\end{array}$ \\
\hline $\begin{array}{l}\text { Blood-Gas Modelling for } \\
\text { Artificially Ventilated Patients } \\
\text { Using Interval Type-2 Fuzzy Logic } \\
\text { System }\end{array}$ & $\begin{array}{l}\text { Siti Hazurah Indera- } \\
\text { Putera, Mahdi Mahfouf, } \\
\text { and G.H. Mills }\end{array}$ & $\begin{array}{l}\text { DOI: } 10.1007 / 978-3-319- \\
\text { 32703-7_193 } \\
\text { pp. 988-993 }\end{array}$ & $\begin{array}{l}\text { DOI: } 10.1007 / 978-3-319- \\
\text { 32703-7_194 } \\
\text { pp. 994-999 }\end{array}$ \\
\hline $\begin{array}{l}\text { A Matlab Tool to Support } \\
\text { Systematic Literature Review with } \\
\text { Meta-Analysis }\end{array}$ & $\begin{array}{l}\text { Pasqua Meccariello, } \\
\text { Rosanna Castaldo, Luis } \\
\text { Montesinos, Eugenio } \\
\text { Guglielmelli, and Leandro } \\
\text { Pecchia }\end{array}$ & $\begin{array}{l}\text { DOI: } 10.1007 / 978-3-319- \\
\text { 32703-7_194 } \\
\text { pp. 994-996 }\end{array}$ & $\begin{array}{l}\text { DOI: } 10.1007 / 978-3-319- \\
32703-7 \_195 \\
\text { pp. } 1000-1002\end{array}$ \\
\hline $\begin{array}{l}\text { Early Assessment of the Added } \\
\text { Value of FDG-PET/CT and MRI- } \\
\text { DWI in Monitoring Treatment } \\
\text { Response of Stage III Non-Small } \\
\text { Cell Lung Cancer }\end{array}$ & $\begin{array}{l}\text { Klaske R. Siegersma, J. } \\
\text { Marjan Hummel, Henk } \\
\text { Broekhuizen, and Lioe-Fee } \\
\text { de Geus-Oei }\end{array}$ & $\begin{array}{l}\text { DOI: } 10.1007 / 978-3-319- \\
\text { 32703-7_195 } \\
\text { pp. 997-999 }\end{array}$ & $\begin{array}{l}\text { DOI: } 10.1007 / 978-3-319- \\
32703-7 \_196 \\
\text { pp. 1003-1005 }\end{array}$ \\
\hline $\begin{array}{l}\text { Hospital Based HTA - } \\
\text { Implementation for the Czech } \\
\text { Republic }\end{array}$ & $\begin{array}{l}\text { Ivana Kubátová and } \\
\text { Veronika Matloňová }\end{array}$ & $\begin{array}{l}\text { DOI: } 10.1007 / 978-3-319- \\
32703-7 \_196 \\
\text { pp. } 1000-1003\end{array}$ & $\begin{array}{l}\text { DOI: } 10.1007 / 978-3-319- \\
32703-7 \_197 \\
\text { pp. } 1006-1009\end{array}$ \\
\hline $\begin{array}{l}\text { Integrated HTA and FMECA } \\
\text { Methodology for the Evaluation of } \\
\text { Robotic Surgery }\end{array}$ & $\begin{array}{l}\text { Roberto Miniati, Fabrizio } \\
\text { Dori, Francesco Frosini, } \\
\text { Paolo Avezzano, Saverio } \\
\text { Grillone, Ernesto Iadanza, } \\
\text { Stefano Vezzosi, and } \\
\text { Andrea Belardinelli } \\
\end{array}$ & $\begin{array}{l}\text { DOI: } 10.1007 / 978-3-319- \\
32703-7 \_197 \\
\text { pp. } 1004-1007\end{array}$ & $\begin{array}{l}\text { DOI: } 10.1007 / 978-3-319- \\
32703-7 \_198 \\
\text { pp. } 1010-1013\end{array}$ \\
\hline $\begin{array}{l}\text { The Potential Power of sub-Saharan } \\
\text { Africa Professional Associations for } \\
\text { Biomedical/Clinical Engineering } \\
\text { Professionals }\end{array}$ & Anna M.E. Worm & $\begin{array}{l}\text { DOI: } 10.1007 / 978-3-319- \\
\text { 32703-7_198 } \\
\text { pp. } 1008-1012\end{array}$ & $\begin{array}{l}\text { DOI: } 10.1007 / 978-3-319- \\
32703-7 \_199 \\
\text { pp. 1014-1018 }\end{array}$ \\
\hline $\begin{array}{l}\text { Risk Management Process on a New } \\
\text { Microwave Thermal Ablation } \\
\text { Device: Assessment and Follow Up }\end{array}$ & $\begin{array}{l}\text { Ernesto Iadanza, Cosimo } \\
\text { Ignesti, Roberto Miniati, } \\
\text { and Alessio Luschi }\end{array}$ & $\begin{array}{l}\text { DOI: } 10.1007 / 978-3-319- \\
32703-7 \_199 \\
\text { pp. 1013-1017 }\end{array}$ & $\begin{array}{l}\text { DOI: } 10.1007 / 978-3-319- \\
32703-7 \_200 \\
\text { pp. 1019-1023 }\end{array}$ \\
\hline $\begin{array}{l}\text { Root Cause Analysis Combined } \\
\text { with Human Factors Engineering } \\
\text { Tools for Adverse Events } \\
\text { Investigation in Healthcare }\end{array}$ & $\begin{array}{l}\text { Victor Hugo Batista } \\
\text { Tsukahara and Saide J. } \\
\text { Calil }\end{array}$ & $\begin{array}{l}\text { DOI: } 10.1007 / 978-3-319- \\
32703-7 \_200 \\
\text { pp. } 1018-1021\end{array}$ & $\begin{array}{l}\text { DOI: } 10.1007 / 978-3-319- \\
32703-7 \_201 \\
\text { pp. 1024-1027 }\end{array}$ \\
\hline $\begin{array}{l}\text { Crisis, What Crisis? How Clinical } \\
\text { Engineers Will Solve the Billion } \\
\text { Dollar Healthcare Funding Gap }\end{array}$ & Dan J. Clark & $\begin{array}{l}\text { DOI: } 10.1007 / 978-3-319- \\
32703-7 \_201 \\
\text { pp. } 1022-1023\end{array}$ & $\begin{array}{l}\text { DOI: } 10.1007 / 978-3-319- \\
32703-7 \_202 \\
\text { pp. } 1028-1029\end{array}$ \\
\hline $\begin{array}{l}\text { Electroporation-Enhanced } \\
\text { Transdermal Delivery of Patent } \\
\text { Blue Using Green Skin Pore Device }\end{array}$ & $\begin{array}{l}\text { Barbara Zorec, Jure } \\
\text { Jelenc, Damijan } \\
\text { Miklavčič, and Nataša } \\
\text { Pavšelj }\end{array}$ & $\begin{array}{l}\text { DOI: } 10.1007 / 978-3-319- \\
32703-7 \_202 \\
\text { pp. 1024-1027 }\end{array}$ & $\begin{array}{l}\text { DOI: } 10.1007 / 978-3-319- \\
32703-7 \_203 \\
\text { pp. } 1030-1033\end{array}$ \\
\hline $\begin{array}{l}\text { A Novel Approach to Improve the } \\
\text { Technical Maintenance of } \\
\text { Biomedical Equipment }\end{array}$ & $\begin{array}{l}\text { Daniele Bibbo, Maurizio } \\
\text { Schmid, Andrea Scorza, } \\
\text { Salvatore Andrea Sciuto, } \\
\text { and Silvia Conforto }\end{array}$ & $\begin{array}{l}\text { DOI: } 10.1007 / 978-3-319- \\
32703-7 \_203 \\
\text { pp. } 1028-1033\end{array}$ & $\begin{array}{l}\text { DOI: } 10.1007 / 978-3-319- \\
32703-7 \_204 \\
\text { pp. } 1034-1039\end{array}$ \\
\hline
\end{tabular}




\begin{tabular}{|c|c|c|c|}
\hline $\begin{array}{l}\text { Developing a Strategic Radiation } \\
\text { Protection Agenda with the } \\
\text { Installation a Dose Monitoring } \\
\text { Software }\end{array}$ & $\begin{array}{l}\text { Charilaos Apostolidis, } \\
\text { Vasileios Ftikas, Dimitrios } \\
\text { Apostolidis, D. Ftikas, and } \\
\text { Aspasia Papadouli }\end{array}$ & $\begin{array}{l}\text { DOI: } 10.1007 / 978-3-319- \\
32703-7 \_204 \\
\text { pp. 1034-1037 }\end{array}$ & $\begin{array}{l}\text { DOI: } 10.1007 / 978-3-319- \\
32703-7 \_205 \\
\text { pp. } 1040-1043\end{array}$ \\
\hline $\begin{array}{l}\text { A Novel Generic Algorithm for } \\
\text { Robust Physiological Signal } \\
\text { Classification }\end{array}$ & $\begin{array}{l}\text { Shadi Mahdiani, Jukka } \\
\text { Vanhala, and Jari Viik }\end{array}$ & $\begin{array}{l}\text { DOI: } 10.1007 / 978-3-319- \\
32703-7 \_205 \\
\text { pp. 1038-1043 }\end{array}$ & $\begin{array}{l}\text { DOI: } 10.1007 / 978-3-319- \\
\text { 32703-7_206 } \\
\text { pp. 1044-1049 }\end{array}$ \\
\hline $\begin{array}{l}\text { HTA of a Large Tablet System in } \\
\text { Digital Pathology }\end{array}$ & $\begin{array}{l}\text { Daniele Giansanti, } \\
\text { Giovanni Maccioni, Marco } \\
\text { Pochini, and Maria } \\
\text { Rosaria Giovagnoli }\end{array}$ & $\begin{array}{l}\text { DOI: } 10.1007 / 978-3-319- \\
32703-7 \_206 \\
\text { pp. } 1044-1047\end{array}$ & $\begin{array}{l}\text { DOI: } 10.1007 / 978-3-319- \\
32703-7 \_207 \\
\text { pp. } 1050-1053\end{array}$ \\
\hline $\begin{array}{l}\text { Haemostasis in Minimal Invasive } \\
\text { Gynaecological Surgery Energies: } \\
\text { Technical Aspects, Safety and } \\
\text { Efficacy }\end{array}$ & $\begin{array}{l}\text { Vasilios Tanos, Marios } \\
\text { Neofytou, and } \\
\text { Constantinos Pattichis }\end{array}$ & $\begin{array}{l}\text { DOI: } 10.1007 / 978-3-319- \\
32703-7 \_207 \\
\text { pp. 1048-1051 }\end{array}$ & $\begin{array}{l}\text { DOI: } 10.1007 / 978-3-319- \\
32703-7 \_208 \\
\text { pp. 1054-1057 }\end{array}$ \\
\hline $\begin{array}{l}\text { The Accuracy of Temperature } \\
\text { Monitoring of the Incubator for } \\
\text { Newborns }\end{array}$ & $\begin{array}{l}\text { Saleh S. Altayyar, } \\
\text { Mahmoud Omer Mohamed } \\
\text { Ali, and Eltahir } \\
\text { Mohammed Hussein }\end{array}$ & $\begin{array}{l}\text { DOI: } 10.1007 / 978-3-319- \\
32703-7 \_208 \\
\text { pp. 1052-1054 }\end{array}$ & $\begin{array}{l}\text { DOI: } 10.1007 / 978-3-319- \\
32703-7 \_209 \\
\text { pp. } 1058-1060\end{array}$ \\
\hline $\begin{array}{l}\text { Comparing the EU and USA } \\
\text { Medical Devices Vigilance Systems } \\
\text { Transparency }\end{array}$ & $\begin{array}{l}\text { Panagiotis Malataras and } \\
\text { Nicolas Pallikarakis }\end{array}$ & $\begin{array}{l}\text { DOI: } 10.1007 / 978-3-319- \\
32703-7 \_209 \\
\text { pp. 1055-1059 }\end{array}$ & $\begin{array}{l}\text { DOI: } 10.1007 / 978-3-319- \\
32703-7 \_210 \\
\text { pp. 1061-1065 }\end{array}$ \\
\hline $\begin{array}{l}\text { A Computational Model of the } \\
\text { Cerebellum to Simulate Cortical } \\
\text { Degeneration During a Pavlovian } \\
\text { Associative Paradigm }\end{array}$ & $\begin{array}{l}\text { Alice Geminiani, Alberto } \\
\text { Antonietti, Claudia } \\
\text { Casellato, Egidio } \\
\text { D'Angelo, and Alessandra } \\
\text { Pedrocchi }\end{array}$ & $\begin{array}{l}\text { DOI: } 10.1007 / 978-3-319- \\
32703-7 \_210 \\
\text { pp. 1063-1068 }\end{array}$ & $\begin{array}{l}\text { DOI: } 10.1007 / 978-3-319- \\
32703-7 \_211 \\
\text { pp. 1069-1074 }\end{array}$ \\
\hline $\begin{array}{l}\text { Reliability of Rapid TMS Stimulus- } \\
\text { Response Curves During Tibialis } \\
\text { Anterior Contractions on Healthy } \\
\text { Elderly }\end{array}$ & $\begin{array}{l}\text { Elisabetta Peri, Vera } \\
\text { Maria Colombo, Emilia } \\
\text { Ambrosini, Mark van de } \\
\text { Ruit, Michael J. Grey, } \\
\text { Marco Monticone, } \\
\text { Alessandra Pedrocchi, } \\
\text { Giancarlo Ferrigno, and } \\
\text { Simona Ferrante }\end{array}$ & $\begin{array}{l}\text { DOI: } 10.1007 / 978-3-319- \\
32703-7 \_211 \\
\text { pp. 1069-1074 }\end{array}$ & $\begin{array}{l}\text { DOI: } 10.1007 / 978-3-319- \\
32703-7 \_212 \\
\text { pp. 1075-1080 }\end{array}$ \\
\hline $\begin{array}{l}\text { Metallic Stents: Biomechanical } \\
\text { Analysis and In Vivo Investigation } \\
\text { of the Vessel Inflammatory } \\
\text { Response }\end{array}$ & $\begin{array}{l}\text { Konstantinos Kapnisis, } \\
\text { Costas Pitsillides, } \\
\text { Marianna Prokopi, G. } \\
\text { Constantinides, Daniel } \\
\text { Cristea, Daniel Munteanu, } \\
\text { Brigitta Brott, Peter } \\
\text { Anderson, Jack Lemons, } \\
\text { and Andreas Anayiotos } \\
\end{array}$ & $\begin{array}{l}\text { DOI: } 10.1007 / 978-3-319- \\
32703-7 \_212 \\
\text { pp. } 1075-1078\end{array}$ & $\begin{array}{l}\text { DOI: } 10.1007 / 978-3-319- \\
32703-7 \_213 \\
\text { pp. 1081-1084 }\end{array}$ \\
\hline $\begin{array}{l}\text { Stacking of Network Based } \\
\text { Classifiers with Application in } \\
\text { Breast Cancer Classification }\end{array}$ & $\begin{array}{l}\text { Stelios Sfakianakis, } \\
\text { Ekaterini S. Bei, and } \\
\text { Michalis Zervakis }\end{array}$ & $\begin{array}{l}\text { DOI: } 10.1007 / 978-3-319- \\
32703-7 \_213 \\
\text { pp. 1079-1082 }\end{array}$ & $\begin{array}{l}\text { DOI: } 10.1007 / 978-3-319- \\
32703-7 \_214 \\
\text { pp. } 1085-1090\end{array}$ \\
\hline $\begin{array}{l}\text { Towards 5G Health for Medical } \\
\text { Video Streaming over Small Cells }\end{array}$ & $\begin{array}{l}\text { Nada Y. Philip and Ikram } \\
\text { U. Rehman }\end{array}$ & $\begin{array}{l}\text { DOI: } 10.1007 / 978-3-319- \\
32703-7 \_214 \\
\text { pp. 1085-1092 }\end{array}$ & $\begin{array}{l}\text { DOI: } 10.1007 / 978-3-319- \\
32703-7 \_215 \\
\text { pp. 1093-1098 }\end{array}$ \\
\hline $\begin{array}{l}\text { Dynamic Network Adaptation for } \\
\text { Real-Time Medical Video } \\
\text { Communication }\end{array}$ & $\begin{array}{l}\text { Zinonas } C . \text { Antoniou, } \\
\text { Andreas S. Panayides, } \\
\text { Marios Pantziaris, } \\
\text { Anthony G. } \\
\text { Constantinides, } \\
\text { Constantinos S. Pattichis, } \\
\text { and Marios S. Pattichis }\end{array}$ & $\begin{array}{l}\text { DOI: } 10.1007 / 978-3-319- \\
32703-7 \_215 \\
\text { pp. 1093-1098 }\end{array}$ & $\begin{array}{l}\text { DOI: } 10.1007 / 978-3-319- \\
32703-7 \_216 \\
\text { pp. 1099-1104 }\end{array}$ \\
\hline
\end{tabular}




\begin{tabular}{|c|c|c|c|}
\hline $\begin{array}{l}\text { Video Analytics for Activity } \\
\text { Recognition in Indoor Environments } \\
\text { Using Fisheye Cameras }\end{array}$ & $\begin{array}{l}\text { Ilias Maglogiannis and } \\
\text { Konstantinos Delibasis }\end{array}$ & $\begin{array}{l}\text { DOI: } 10.1007 / 978-3-319- \\
32703-7 \_216 \\
\text { pp. 1099-1104 }\end{array}$ & $\begin{array}{l}\text { DOI: } 10.1007 / 978-3-319- \\
32703-7 \_217 \\
\text { pp. } 1105-1110\end{array}$ \\
\hline $\begin{array}{l}\text { Secure and Compact Image-Based } \\
\text { Storage Format for DICOM } \\
\text { Multiframe Echocardiogram Video }\end{array}$ & $\begin{array}{l}\text { Alvaro Alesanco, Oscar J. } \\
\text { Rubio, Eva Cavero, Jorge } \\
\text { Sancho, and Jose Garcia }\end{array}$ & $\begin{array}{l}\text { DOI: } 10.1007 / 978-3-319- \\
32703-7 \_217 \\
\text { pp. } 1105-1110\end{array}$ & $\begin{array}{l}\text { DOI: } 10.1007 / 978-3-319- \\
32703-7 \_218 \\
\text { pp. } 1111-1116\end{array}$ \\
\hline $\begin{array}{l}\text { Biological Data Tracing and Pattern } \\
\text { Recognition in Real-time }\end{array}$ & $\begin{array}{l}\text { W. Daniel Scherz, Luis } \\
\text { Miguel Soria Morillo, and } \\
\text { Ralf Seepold }\end{array}$ & $\begin{array}{l}\text { DOI: } 10.1007 / 978-3-319- \\
32703-7 \_218 \\
\text { pp. } 1113-1118\end{array}$ & $\begin{array}{l}\text { DOI: } 10.1007 / 978-3-319- \\
\text { 32703-7_219 } \\
\text { pp. 1119-1124 }\end{array}$ \\
\hline $\begin{array}{l}\text { Multi-dimensional } \\
\text { Kineticardiography a New } \\
\text { Approach for Wearable Cardiac } \\
\text { Monitoring Through Body } \\
\text { Acceleration Recordings }\end{array}$ & $\begin{array}{l}\text { Pierre-François Migeotte, } \\
\text { Vivana Mucci, Quentin } \\
\text { Delière, Laurent Lejeune, } \\
\text { and Philippe van de Borne }\end{array}$ & $\begin{array}{l}\text { DOI: } 10.1007 / 978-3-319- \\
32703-7 \_219 \\
\text { pp. 1119-1124 }\end{array}$ & $\begin{array}{l}\text { DOI: } 10.1007 / 978-3-319- \\
32703-7 \_220 \\
\text { pp. } 1125-1130\end{array}$ \\
\hline $\begin{array}{l}\text { Telemedicine and mHealth System } \\
\text { for Complex Management in T1DM } \\
\text { and T2DM Patients: Results of } 6 \\
\text { Months Study }\end{array}$ & $\begin{array}{l}\text { Fedor Lehocki and Tomas } \\
\text { Bacigal }\end{array}$ & $\begin{array}{l}\text { DOI: } 10.1007 / 978-3-319- \\
32703-7 \_220 \\
\text { pp. } 1125-1130\end{array}$ & $\begin{array}{l}\text { DOI: } 10.1007 / 978-3-319- \\
32703-7 \_221 \\
\text { pp. } 1131-1136\end{array}$ \\
\hline $\begin{array}{l}\text { Mid-Term Prediction of Blood } \\
\text { Glucose from Continuous Glucose } \\
\text { Sensors, Meal Information and } \\
\text { Administered Insulin }\end{array}$ & $\begin{array}{l}\text { Iván Contreras and Josep } \\
\text { Vehi }\end{array}$ & $\begin{array}{l}\text { DOI: 10.1007/978-3-319- } \\
\text { 32703-7_221 } \\
\text { pp. 1131-1137 }\end{array}$ & $\begin{array}{l}\text { DOI: } 10.1007 / 978-3-319- \\
32703-7 \_222 \\
\text { pp. } 1137-1143\end{array}$ \\
\hline $\begin{array}{l}\text { Optimal Short Distance Electrode } \\
\text { Locations for Impedance } \\
\text { Pneumography Measurement from } \\
\text { the Frontal Thoracic Area }\end{array}$ & $\begin{array}{l}\text { Vala Jeyhani, Tiina } \\
\text { Vuorinen, Kai Noponen, } \\
\text { Matti Mäntysalo, and Antti } \\
\text { Vehkaoja }\end{array}$ & $\begin{array}{l}\text { DOI: } 10.1007 / 978-3-319- \\
32703-7 \_222 \\
\text { pp. } 1138-1143\end{array}$ & $\begin{array}{l}\text { DOI: } 10.1007 / 978-3-319- \\
32703-7 \_223 \\
\text { pp. } 1144-1149\end{array}$ \\
\hline $\begin{array}{l}\text { Design and Development of a } \\
\text { Mobile Application to Explore } \\
\text { Cognitive Skills in Parkinson's } \\
\text { Disease Patients }\end{array}$ & $\begin{array}{l}\text { Jorge Cancela, Samanta } \\
\text { Villanueva Mascato, } \\
\text { Giovanni Gentile, Manuela } \\
\text { Giglio, Roberta Biundo, } \\
\text { Maria Teresa Arredondo } \\
\text { Waldmeyer, and Angelo } \\
\text { Antonini }\end{array}$ & $\begin{array}{l}\text { DOI: } 10.1007 / 978-3-319- \\
32703-7 \_223 \\
\text { pp. } 1144-1149\end{array}$ & $\begin{array}{l}\text { DOI: } 10.1007 / 978-3-319- \\
32703-7 \_224 \\
\text { pp. } 1150-1155\end{array}$ \\
\hline $\begin{array}{l}\text { Influence of Stress in Driving } \\
\text { Behaviour }\end{array}$ & $\begin{array}{l}\text { Emre Yay, Natividad } \\
\text { Martinez Madrid, and } \\
\text { Juan Antonio Ortega } \\
\text { Ramirez }\end{array}$ & $\begin{array}{l}\text { DOI: } 10.1007 / 978-3-319- \\
32703-7 \_224 \\
\text { pp. } 1150-1155\end{array}$ & $\begin{array}{l}\text { DOI: } 10.1007 / 978-3-319- \\
32703-7 \_225 \\
\text { pp. } 1156-1161\end{array}$ \\
\hline $\begin{array}{l}\text { Recommendation System: A } \\
\text { Contribution to Glycaemia } \\
\text { Excursion Identification }\end{array}$ & $\begin{array}{l}\text { Lenka Lhotska, Miroslav } \\
\text { Bursa, Michal Huptych, } \\
\text { and Katerina Stechova }\end{array}$ & $\begin{array}{l}\text { DOI: } 10.1007 / 978-3-319- \\
32703-7 \_225 \\
\text { pp. } 1156-1160\end{array}$ & $\begin{array}{l}\text { DOI: } 10.1007 / 978-3-319- \\
32703-7 \_226 \\
\text { pp. } 1162-1166\end{array}$ \\
\hline $\begin{array}{l}\text { Performance of Algorithms for } \\
\text { Interval Discretization of } \\
\text { Biomedical Signals }\end{array}$ & $\begin{array}{l}\text { Luis Miguel Soria Morillo, } \\
\text { Luis Gonzalez-Abril, and } \\
\text { Juan Antonio Ortega } \\
\text { Ramírez }\end{array}$ & $\begin{array}{l}\text { DOI: } 10.1007 / 978-3-319- \\
32703-7 \_226 \\
\text { pp. } 1161-1167\end{array}$ & $\begin{array}{l}\text { DOI: } 10.1007 / 978-3-319- \\
32703-7 \_227 \\
\text { pp. 1167-1173 }\end{array}$ \\
\hline $\begin{array}{l}\text { LifePal: A Mobile Self- } \\
\text { Management Tool for Supporting } \\
\text { Young People with Autism }\end{array}$ & $\begin{array}{l}\text { Kerry-Louise Skillen, Mark } \\
\text { P. Donnelly, Chris D. } \\
\text { Nugent, and Nichola Booth }\end{array}$ & $\begin{array}{l}\text { DOI: } 10.1007 / 978-3-319- \\
32703-7 \_227 \\
\text { pp. } 1168-1173\end{array}$ & $\begin{array}{l}\text { DOI: } 10.1007 / 978-3-319- \\
32703-7 \_228 \\
\text { pp. 1174-1179 }\end{array}$ \\
\hline $\begin{array}{l}\text { The Evolution of mHealth } \\
\text { Interventions in Heart Failure }\end{array}$ & $\begin{array}{l}\text { Georgia S. Karanasiou, } \\
\text { Evanthia E. Tripoliti, } \\
\text { Fanis G. Kalatzis, } \\
\text { Abdelhamid Errachid, and } \\
\text { Dimitrios I. Fotiadis }\end{array}$ & $\begin{array}{l}\text { DOI: } 10.1007 / 978-3-319- \\
32703-7 \_228 \\
\text { pp. } 1174-1178\end{array}$ & $\begin{array}{l}\text { DOI: } 10.1007 / 978-3-319- \\
32703-7 \_229 \\
\text { pp. 1180-1184 }\end{array}$ \\
\hline $\begin{array}{l}\text { Assessing Pediatrics Patients' } \\
\text { Psychological States from } \\
\text { Biomedical Signals in a Cloud of } \\
\text { Social Robots }\end{array}$ & $\begin{array}{l}\text { Marta Díaz-Boladeras, Cecilio } \\
\text { Angulo, Miquel Domènech, } \\
\text { Jordi Albo-Canals, Núria } \\
\text { Serrallonga, Cristóbal Raya, } \\
\text { and Alex Barco }\end{array}$ & $\begin{array}{l}\text { DOI: } 10.1007 / 978-3-319- \\
\text { 32703-7_229 } \\
\text { pp. 1179-1184 }\end{array}$ & $\begin{array}{l}\text { DOI: } 10.1007 / 978-3-319- \\
32703-7 \_230 \\
\text { pp. 1185-1190 }\end{array}$ \\
\hline
\end{tabular}




\begin{tabular}{|c|c|c|c|}
\hline $\begin{array}{l}\text { Survey on Cardiotocography } \\
\text { Feature Extraction Algorithms for } \\
\text { Foetal Welfare Assessment }\end{array}$ & $\begin{array}{l}\text { Michel Haritopoulos, } \\
\text { Alfredo Illanes, and Asoke } \\
\text { K. Nandi }\end{array}$ & $\begin{array}{l}\text { DOI: } 10.1007 / 978-3-319- \\
32703-7 \_230 \\
\text { pp. } 1187-1192\end{array}$ & $\begin{array}{l}\text { DOI: } 10.1007 / 978-3-319- \\
32703-7 \_231 \\
\text { pp. } 1193-1198\end{array}$ \\
\hline $\begin{array}{l}\text { Intrapartum Fetal Heart Rate } \\
\text { Classification: Cross-Database } \\
\text { Evaluation }\end{array}$ & $\begin{array}{l}\text { Jiř́ Spilka, Václav } \\
\text { Chudáček, Michal } \\
\text { Huptych, Roberto } \\
\text { Leonarduzzi, Patrice Abry, } \\
\text { and Muriel Doret }\end{array}$ & $\begin{array}{l}\text { DOI: } 10.1007 / 978-3-319- \\
32703-7 \_231 \\
\text { pp. 1193-1198 }\end{array}$ & $\begin{array}{l}\text { DOI: } 10.1007 / 978-3-319- \\
32703-7 \_232 \\
\text { pp. 1199-1204 }\end{array}$ \\
\hline $\begin{array}{l}\text { Advanced Signal Processing } \\
\text { Techniques for CTG Analysis }\end{array}$ & $\begin{array}{l}\text { Maria G. Signorini and } \\
\text { Giovanni Magenes }\end{array}$ & $\begin{array}{l}\text { DOI: } 10.1007 / 978-3-319- \\
32703-7 \_232 \\
\text { pp. } 1199-1204\end{array}$ & $\begin{array}{l}\text { DOI: } 10.1007 / 978-3-319- \\
32703-7 \_233 \\
\text { pp. } 1205-1210\end{array}$ \\
\hline $\begin{array}{l}\text { Least Squares Support Vector } \\
\text { Machines for FHR Classification } \\
\text { and Assessing the pH Based } \\
\text { Categorization }\end{array}$ & $\begin{array}{l}\text { Chrysostomos D. Stylios, } \\
\text { George Georgoulas, } \\
\text { Petros Karvelis, Jiri } \\
\text { Spilka, Václav Chudáček, } \\
\text { and Lenka Lhotska }\end{array}$ & $\begin{array}{l}\text { DOI: } 10.1007 / 978-3-319- \\
32703-7 \_233 \\
\text { pp. } 1205-1209\end{array}$ & $\begin{array}{l}\text { DOI: } 10.1007 / 978-3-319- \\
32703-7 \_234 \\
\text { pp. } 1211-1215\end{array}$ \\
\hline $\begin{array}{l}\text { Analysis of Intrapartum Fetal Heart } \\
\text { Rate According to Umbilical } \\
\text { Arterial pH and Age of Mother }\end{array}$ & $\begin{array}{l}\text { Nathalie T.E. Gayraud, } \\
\text { Marina Argiri, and George } \\
\text { Manis }\end{array}$ & $\begin{array}{l}\text { DOI: } 10.1007 / 978-3-319- \\
32703-7 \_234 \\
\text { pp. } 1210-1215\end{array}$ & $\begin{array}{l}\text { DOI: } 10.1007 / 978-3-319- \\
32703-7 \_235 \\
\text { pp. } 1216-1221\end{array}$ \\
\hline $\begin{array}{l}\text { GameUp: Exergames for Mobility - } \\
\text { A Project to Keep Elderly Active }\end{array}$ & $\begin{array}{l}\text { Ellen Brox, Stathis Th. } \\
\text { Konstantinidis, Gunn } \\
\text { Evertsen, Luis Fernandez- } \\
\text { Luque, Antonio } \\
\text { Remartinez, Peter Oesch, } \\
\text { and Anton Civit }\end{array}$ & $\begin{array}{l}\text { DOI: } 10.1007 / 978-3-319- \\
32703-7 \_235 \\
\text { pp. 1219-1224 }\end{array}$ & $\begin{array}{l}\text { DOI: } 10.1007 / 978-3-319- \\
32703-7 \_236 \\
\text { pp. } 1225-1230\end{array}$ \\
\hline $\begin{array}{l}\text { Indoor Location IoT Analytics "in } \\
\text { the wild": Active and Healthy } \\
\text { Ageing Cases }\end{array}$ & $\begin{array}{l}\text { E.I. Konstantinidis, A.S. } \\
\text { Billis, L. Plotegher, G. } \\
\text { Conti, and P.D. Bamidis }\end{array}$ & $\begin{array}{l}\text { DOI: } 10.1007 / 978-3-319- \\
32703-7 \_236 \\
\text { pp. } 1225-1230\end{array}$ & $\begin{array}{l}\text { DOI: } 10.1007 / 978-3-319- \\
32703-7 \_237 \\
\text { pp. } 1231-1236\end{array}$ \\
\hline $\begin{array}{l}\text { Indoor Localisation Through Object } \\
\text { Detection on Real-Time Video } \\
\text { Implementing a Single Wearable } \\
\text { Camera }\end{array}$ & $\begin{array}{l}\text { Colin Shewell, Chris } \\
\text { Nugent, Mark Donnelly, } \\
\text { and Haiying Wang }\end{array}$ & $\begin{array}{l}\text { DOI: } 10.1007 / 978-3-319- \\
32703-7 \_237 \\
\text { pp. } 1231-1236\end{array}$ & $\begin{array}{l}\text { DOI: } 10.1007 / 978-3-319- \\
32703-7 \_238 \\
\text { pp. } 1237-1242\end{array}$ \\
\hline $\begin{array}{l}\text { Monitoring of Compliance on an } \\
\text { Individual Treatment Through } \\
\text { Mobile Innovations }\end{array}$ & $\begin{array}{l}\text { Athanasios Anastasiou, } \\
\text { Kostas Giokas, and } \\
\text { Dimitris Koutsouris }\end{array}$ & $\begin{array}{l}\text { DOI: } 10.1007 / 978-3-319- \\
32703-7 \_238 \\
\text { pp. } 1237-1242\end{array}$ & $\begin{array}{l}\text { DOI: } 10.1007 / 978-3-319- \\
32703-7 \_239 \\
\text { pp. } 1243-1248\end{array}$ \\
\hline $\begin{array}{l}\text { Presence Detection from Smart } \\
\text { Home Motion Sensor Datasets: A } \\
\text { Model }\end{array}$ & $\begin{array}{l}\text { Edewede Oriwoh and } M . \\
\text { Conrad }\end{array}$ & $\begin{array}{l}\text { DOI: } 10.1007 / 978-3-319- \\
32703-7 \_239 \\
\text { pp. } 1243-1249\end{array}$ & $\begin{array}{l}\text { DOI: } 10.1007 / 978-3-319- \\
32703-7 \_240 \\
\text { pp. } 1249-1255\end{array}$ \\
\hline $\begin{array}{l}\text { Chest Electrical Impedance } \\
\text { Tomography and Its Clinical } \\
\text { Applications }\end{array}$ & $\begin{array}{l}\text { Zhanqi Zhao and Knut } \\
\text { Möller }\end{array}$ & $\begin{array}{l}\text { DOI: } 10.1007 / 978-3-319- \\
32703-7 \_240 \\
\text { pp. } 1253-1257\end{array}$ & $\begin{array}{l}\text { DOI: } 10.1007 / 978-3-319- \\
32703-7 \_241 \\
\text { pp. } 1259-1263\end{array}$ \\
\hline $\begin{array}{l}\text { Center of Ventilation-Methods of } \\
\text { Calculation Using Electrical } \\
\text { Impedance Tomography and the } \\
\text { Influence of Image Segmentation }\end{array}$ & $\begin{array}{l}\text { Vladimir Sobota and Karel } \\
\text { Roubik }\end{array}$ & $\begin{array}{l}\text { DOI: } 10.1007 / 978-3-319- \\
32703-7 \_241 \\
\text { pp. } 1258-1263\end{array}$ & $\begin{array}{l}\text { DOI: } 10.1007 / 978-3-319- \\
32703-7 \_242 \\
\text { pp. } 1264-1269\end{array}$ \\
\hline $\begin{array}{l}\text { Impact of Heart Rate on Ventilation } \\
\text { and Pulmonary Perfusion } \\
\text { Associated Impedance Changes }\end{array}$ & $\begin{array}{l}\text { Sabine Krueger-Ziolek, } \\
\text { Zhanqi Zhao, Benjamin } \\
\text { Schullcke, Bo Gong, and } \\
\text { Knut Moeller }\end{array}$ & $\begin{array}{l}\text { DOI: } 10.1007 / 978-3-319- \\
32703-7 \_242 \\
\text { pp. } 1264-1269\end{array}$ & $\begin{array}{l}\text { DOI: } 10.1007 / 978-3-319- \\
32703-7 \_243 \\
\text { pp. } 1270-1275\end{array}$ \\
\hline $\begin{array}{l}\text { EIT Image Reconstruction with } \\
\text { Discrete Cosine Transform }\end{array}$ & $\begin{array}{l}\text { Benjamin Schullcke, Bo } \\
\text { Gong, Sabine Krueger- } \\
\text { Ziolek, and Knut Moeller }\end{array}$ & $\begin{array}{l}\text { DOI: } 10.1007 / 978-3-319- \\
32703-7 \_243 \\
\text { pp. } 1270-1273\end{array}$ & $\begin{array}{l}\text { DOI: } 10.1007 / 978-3-319- \\
32703-7 \_244 \\
\text { pp. } 1276-1279\end{array}$ \\
\hline $\begin{array}{l}\text { EIT Imaging Regularization Based } \\
\text { on Spectral Graph Wavelets }\end{array}$ & $\begin{array}{l}\text { Bo Gong, Benjamin } \\
\text { Schullcke, Sabine Krueger- } \\
\text { Ziolek, and Knut Moeller }\end{array}$ & $\begin{array}{l}\text { DOI: } 10.1007 / 978-3-319- \\
32703-7 \_244 \\
\text { pp. } 1274-1278\end{array}$ & $\begin{array}{l}\text { DOI: } 10.1007 / 978-3-319- \\
32703-7 \_245 \\
\text { pp. } 1280-1284\end{array}$ \\
\hline
\end{tabular}




\begin{tabular}{|c|c|c|c|}
\hline $\begin{array}{l}\text { Gamified Wellbeing for All Ages - } \\
\text { How Technology and Gamification } \\
\text { Can Support Physical and Mental } \\
\text { Wellbeing in the Ageing Society }\end{array}$ & $\begin{array}{l}\text { Lucia Pannese, David } \\
\text { Wortley, and Antonio } \\
\text { Ascolese }\end{array}$ & $\begin{array}{l}\text { DOI: } 10.1007 / 978-3-319- \\
32703-7 \_245 \\
\text { pp. 1281-1285 }\end{array}$ & $\begin{array}{l}\text { DOI: } 10.1007 / 978-3-319- \\
32703-7 \_246 \\
\text { pp. } 1287-1291\end{array}$ \\
\hline $\begin{array}{l}\text { Evaluation of Neurofeedback on } \\
\text { ADHD Using Mobile Health } \\
\text { Technologies }\end{array}$ & $\begin{array}{l}\text { Niki Pandria, Dimitris } \\
\text { Spachos, and Panagiotis } \\
\text { D. Bamidis }\end{array}$ & $\begin{array}{l}\text { DOI: } 10.1007 / 978-3-319- \\
32703-7 \_246 \\
\text { pp. } 1286-1290\end{array}$ & $\begin{array}{l}\text { DOI: } 10.1007 / 978-3-319- \\
32703-7 \_247 \\
\text { pp. } 1292-1296\end{array}$ \\
\hline $\begin{array}{l}\text { Recognizing Emotion from Blood } \\
\text { Volume Pulse and Skin } \\
\text { Conductance Sensor Using Machine } \\
\text { Learning Algorithms }\end{array}$ & $\begin{array}{l}\text { Ali Mehmood Khan and } \\
\text { Michael Lawo }\end{array}$ & $\begin{array}{l}\text { DOI: } 10.1007 / 978-3-319- \\
32703-7 \_247 \\
\text { pp. 1291-1297 }\end{array}$ & $\begin{array}{l}\text { DOI: } 10.1007 / 978-3-319- \\
32703-7 \_248 \\
\text { pp. } 1297-1303\end{array}$ \\
\hline $\begin{array}{l}\text { Speech and Language Support } \\
\text { System for Children with Hearing } \\
\text { Impairment }\end{array}$ & $\begin{array}{l}\text { Efthyvoulos Kyriacou, } \\
\text { Rafail Mavrocheilos, } \\
\text { Chrysovalantis Nikolaou, } \\
\text { Marina Charalambous, } \\
\text { George Hadjichristofi, } \\
\text { Elena Matziari, and Maria } \\
\text { Ioannou }\end{array}$ & $\begin{array}{l}\text { DOI: } 10.1007 / 978-3-319- \\
32703-7 \_248 \\
\text { pp. 1298-1302 }\end{array}$ & $\begin{array}{l}\text { DOI: } 10.1007 / 978-3-319- \\
32703-7 \_249 \\
\text { pp. 1304-1308 }\end{array}$ \\
\hline $\begin{array}{l}\text { Online Decision Support for Speech } \\
\& \text { Language Pathology Assessment } \\
\text { and Rehabilitation of Individuals } \\
\text { with Multiple Needs }\end{array}$ & $\begin{array}{l}\text { Voula C. Georgopoulos, } \\
\text { Chrysostomos D. Stylios, } \\
\text { Maria Kambanaros, and } \\
\text { Georgia A. Malandraki }\end{array}$ & $\begin{array}{l}\text { DOI: } 10.1007 / 978-3-319- \\
32703-7 \_249 \\
\text { pp. 1303-1307 }\end{array}$ & $\begin{array}{l}\text { DOI: } 10.1007 / 978-3-319- \\
32703-7 \_250 \\
\text { pp. 1309-1313 }\end{array}$ \\
\hline $\begin{array}{l}\text { Revamped Biomedical Engineering } \\
\text { Curriculum for Future-Ready } \\
\text { Graduates }\end{array}$ & Siew-Lok Toh & $\begin{array}{l}\text { DOI: } 10.1007 / 978-3-319- \\
32703-7 \_250 \\
\text { pp. } 1311-1313\end{array}$ & $\begin{array}{l}\text { DOI: } 10.1007 / 978-3-319- \\
32703-7 \_251 \\
\text { pp. } 1317-1319\end{array}$ \\
\hline $\begin{array}{l}\text { Master Program in BME for } \\
\text { Engineers and Medical Doctors }\end{array}$ & Ákos Jobbágy & $\begin{array}{l}\text { DOI: } 10.1007 / 978-3-319- \\
32703-7 \_251 \\
\text { pp. } 1314-1316\end{array}$ & $\begin{array}{l}\text { DOI: } 10.1007 / 978-3-319- \\
32703-7 \_252 \\
\text { pp. } 1320-1322\end{array}$ \\
\hline $\begin{array}{l}\text { Biomedical Engineering Education } \\
\text { in the Czech Republic }\end{array}$ & $\begin{array}{l}\text { Lenka Lhotska and Jiri } \\
\text { Hozman }\end{array}$ & $\begin{array}{l}\text { DOI: } 10.1007 / 978-3-319- \\
32703-7 \_252 \\
\text { pp. } 1317-1322\end{array}$ & $\begin{array}{l}\text { DOI: } 10.1007 / 978-3-319- \\
32703-7 \_253 \\
\text { pp. } 1323-1328\end{array}$ \\
\hline $\begin{array}{l}\text { The Status of BME Programs in } \\
\text { Latin America }\end{array}$ & $\begin{array}{l}\text { Martha Zequera Díaz and } \\
\text { A.P. Koch }\end{array}$ & $\begin{array}{l}\text { DOI: } 10.1007 / 978-3-319- \\
32703-7 \_253 \\
\text { p. } 1323\end{array}$ & $\begin{array}{l}\text { DOI: } 10.1007 / 978-3-319- \\
32703-7 \_254 \\
\text { p. } 1329\end{array}$ \\
\hline $\begin{array}{l}\text { Biomedical Engineering, Medical } \\
\text { Engineering, and Bioengineering: } \\
\text { Different BME Programmes in } \\
\text { Hong Kong }\end{array}$ & Yong-Ping Zheng & $\begin{array}{l}\text { DOI: } 10.1007 / 978-3-319- \\
32703-7 \_254 \\
\text { p. } 1324\end{array}$ & $\begin{array}{l}\text { DOI: } 10.1007 / 978-3-319- \\
32703-7 \_255 \\
\text { p. } 1330\end{array}$ \\
\hline $\begin{array}{l}\text { Status of Biomedical Engineering } \\
\text { Education in the Asia Pacific }\end{array}$ & $\begin{array}{l}\text { Kang-Ping Lin, Mei-Fen } \\
\text { Chen, Cheng-Lung Tsai, } \\
\text { and Kao Tsai }\end{array}$ & $\begin{array}{l}\text { DOI: } 10.1007 / 978-3-319- \\
32703-7 \_255 \\
\text { pp. } 1325-1328\end{array}$ & $\begin{array}{l}\text { DOI: } 10.1007 / 978-3-319- \\
32703-7 \_256 \\
\text { pp. 1331-1334 }\end{array}$ \\
\hline $\begin{array}{l}\text { Construction of Inflatable Lungs to } \\
\text { Simulate Respiratory Motion in } \\
\text { Myocardial Perfusion Imaging }\end{array}$ & $\begin{array}{l}\text { Antonis Lontos, } \text { A. } \\
\text { Antoniou, I. Chrysanthou, } \\
\text { S. Christofides, O. } \\
\text { Demetriadou, Chr. } \\
\text { Panagidis, Ch. } \\
\text { Yiannakkaras, D. Kaolis, } \\
\text { and Yiannis Parpottas }\end{array}$ & $\begin{array}{l}\text { DOI: } 10.1007 / 978-3-319- \\
32703-7 \_256 \\
\text { pp. } 1331-1335\end{array}$ & $\begin{array}{l}\text { DOI: } 10.1007 / 978-3-319- \\
32703-7 \_257 \\
\text { pp. } 1337-1341\end{array}$ \\
\hline $\begin{array}{l}\text { Current and Future Trends in the } \\
\text { HTA of Medical Devices }\end{array}$ & $\begin{array}{l}\text { Oriana Ciani, Carlo } \\
\text { Federici, and Rosanna } \\
\text { Tarricone }\end{array}$ & $\begin{array}{l}\text { DOI: } 10.1007 / 978-3-319- \\
32703-7 \_257 \\
\text { pp. 1339-1342 }\end{array}$ & $\begin{array}{l}\text { DOI: } 10.1007 / 978-3-319- \\
32703-7 \_258 \\
\text { pp. } 1345-1348\end{array}$ \\
\hline $\begin{array}{l}\text { Real-Time Workload Assessment } \\
\text { Using EEG Signals in Virtual } \\
\text { Reality Environment }\end{array}$ & $\begin{array}{l}\text { Shen Ren, Fabio Babiloni, } \\
\text { Nitish V. Thakor, and } \\
\text { Anastasios Bezerianos }\end{array}$ & $\begin{array}{l}\text { DOI: } 10.1007 / 978-3-319- \\
32703-7 \_258 \\
\text { pp. } 1345-1346\end{array}$ & $\begin{array}{l}\text { DOI: } 10.1007 / 978-3-319- \\
32703-7 \_259 \\
\text { pp. } 1351-1352\end{array}$ \\
\hline
\end{tabular}

